\title{
TOWARDS THE INTERSECTION THEORY ON HURWITZ SPACES
}

\author{
M. E. Kazaryan, S. K. LANdo
}

01.04 .2004

\begin{abstract}
Moduli spaces of algebraic curves and closely related to them Hurwitz spaces, that is, spaces of meromorphic functions on the curves, arise naturally in numerous problems of algebraic geometry and mathematical physics, especially in relationship with the string theory and Gromov-Witten invariants. In particular, the classical Hurwitz problem about enumeration of topologically distinct ramified coverings of the sphere with prescribed ramification type reduces to the study of geometry and topology of these spaces. The cohomology rings of such spaces are complicated even in the simplest cases of rational curves and functions. However, the cohomology classes that are the most important from the point of view of applications (namely, the classes Poincaré dual to the strata of functions with given singularities) can be expressed in terms of relatively simple "basic" classes (which are, in a sense, tautological). The aim of the present paper is to identify these basic classes, to describe relations among them, and to find expressions for the strata in terms of these classes. Our approach is based on R. Thom's theory of universal polynomials of singularities, which has been extended to the case of multisingularities by the first author. Although the general Hurwitz problem still remains open, our approach allows one to achieve a significant progress in its solution, as well as in the understanding of the geometry and topology of Hurwitz spaces.
\end{abstract}

\section{$\S 1$ INTRODUCTION}

1.1. Hurwitz's problem. In [8] A. Hurwitz suggested the following problem: enumerate isomorphism classes of ramified coverings of the 2 -sphere by a surface of genus $g$ having prescribed ramification points in the target sphere and prescribed ramification types over each ramification point. (The formal statement of the problem is given below.) During the last century the problem attracted researchers' attention several times, however this direction of mathematical research became a central one only recently. The explosion of interest to the problem is due first of all to the applications it has found in modern mathematical physics, namely, in the string theory and the theory of Gromov-Witten invariants.

Up to now, the Hurwitz problem does not have a satisfactory solution: known enumerative formulas lead to transparent answers only in some partial cases.

1991 Mathematics Subject Classification. 14H30, 14C17, 14H10.

The research was supported by the RFBR grants (no. 04-01-00762 for the first author and no. 02-01-22004 for the second one). 
In [1], [2] a relationship between ramified coverings of the sphere and intersection theory on moduli spaces of functions on algebraic curves has been established. The very existence of such connection revives hopes for obtaining simple general answers. In [1], [2] the Hurwitz problem was reduced to a problem concerning the geometry of moduli spaces; the latter is of its own interest. Because of the construction in [2] it is closely related to the already classical problem about the geometry of moduli spaces of algebraic curves.

In the present paper we analyze the part of the geometry of Hurwitz spaces directly related to the Hurwitz problem.

1.2. Hurwitz spaces and cohomology classes of strata. We shall consider ramified coverings of the 2 -sphere by surfaces of genus $g$; let $n$ denote the degree of the coverings. In addition to topological coverings, we shall also consider meromorphic functions of degree $n$ on algebraic curves of genus $g$. Of course, from the topological point of view such a function is a ramified covering. Denote by $\mathcal{H}_{g, n}$ the space of such functions possessing the following properties:

- all the poles of the functions are simple, i.e., the meromorphic function has $n$ poles of order 1; moreover, we assume that the poles of each function are numbered;

- the sum of the critical values of the function is 0 .

According to [2] this space is a smooth complex orbifold (or even a smooth variety provided that $g=0$ or $n$ is sufficiently large). It is fibered over the moduli space $\mathcal{M}_{g, n}$ of complex curves of genus $g$ with $n$ marked points: to each function one can associate its underlying curve equipped with the $n$ marked poles.

The space $\mathcal{H}_{g, n}$ possesses a completion denoted by $\overline{\mathcal{H}}_{g, n}$ and consisting of stable meromorphic functions [2], [14]. Its boundary $\overline{\mathcal{H}}_{g, n} \backslash \mathcal{H}_{g, n}$ consists of stable meromorphic functions with a singular underlying curve, the only admissible singularities being nodes (points of double selfintersection). The projection $\mathcal{H}_{g, n} \rightarrow \mathcal{M}_{g, n}$ extends to a projection $\overline{\mathcal{H}}_{g, n} \rightarrow \overline{\mathcal{M}}_{g, n}$ of the completed space to the moduli space of stable curves with marked points. The fibers of this projection are vector spaces since a linear combination of meromorphic functions with poles of order at most one at the marked points is a function of the same kind. Note, however, that generally speaking this projection is not a vector bundle since the dimension of the fiber can vary from one point of the base to another. The fiberwise projectivization $P \overline{\mathcal{H}}_{g, n}$ of the completed Hurwitz space is a compact complex orbifold. This will be our main moduli space, and all the other required spaces will be constructed using it. By some abuse of language, we speak below about varieties and subvarieties, having in mind that our spaces are, in fact, orbifolds and suborbifolds (i.e., locally they look like quotient spaces of the complex disc modulo a finite group action).

Since the variety $P \overline{\mathcal{H}}_{g, n}$ is a projectivization, it carries a natural second cohomology class, namely, the first Chern class of the tautological sheaf, which we denote by $\psi=\psi_{g, n}=c_{1}(\mathcal{O}(1)) \in H^{2}\left(P \overline{\mathcal{H}}_{g, n}\right)$.

On the other hand, this variety contains subvarieties consisting of functions with degenerate ramification. By the Riemann-Hurwitz formula, a generic meromorphic function of degree $n$ on a curve of genus $g$ has $2 n+2 g-2$ points of nondegenerate ramification (both in the source and in the target). The functions with fewer ramification points in the target sphere form the discriminant in the space of functions. One can assign to each ramification point in the target sphere a partition of $n$ which is the (unordered) set of multiplicities of the preimages of this point. It will 
multiplicities of the preimages decreased by one. We denote the closure in $P \overline{\mathcal{H}}_{g, n}$ of the set functions having ramification of prescribed type by $\sigma_{\alpha_{1}, \ldots, \alpha_{c}}$; the subscript here consists of reduced partitions over the points of degenerate ramification. These subvarieties are called the strata of the discriminant. Thus, $\sigma_{2^{1}} \subset P \overline{\mathcal{H}}_{g, n}$ denotes the caustic, i.e., the stratum consisting of functions with two ramification points in the preimage glued together, and $\sigma_{1^{2}}$ is the Maxwell stratum whose open part consists of functions taking the same value at two distinct critical points. The caustic and the Maxwell stratum are the only strata of (complex) codimension one. Similarly, the stratum $\sigma_{2^{1}, 1^{2}}$, of codimension 2 , is the closure of the set of functions with two degenerate critical values, with a ramification point of multiplicity 3 over one of them, and two simple ramification points over the other one, and so on. The number of nondegenerate critical points (corresponding to the reduced partition $1^{1}$ ) for a generic function in a given stratum can be easily calculated by means of the Riemann-Hurwitz formula. For the sake of simplicity of notation we do not include these nondegenerate reduced partitions, as well as the values $g$ and $n$, in the notation for the strata.

Each stratum is a complex subvariety of pure codimension in $P \overline{\mathcal{H}}_{g, n}$, whence it determines, by Poincaré duality, a homogeneous class in the cohomology ring

$$
H^{*}\left(P \overline{\mathcal{H}}_{g, n}\right)=H^{*}\left(P \overline{\mathcal{H}}_{g, n}, \mathbb{Q}\right) \text {. }
$$

(Everywhere below we are interested in cohomology with rational coefficients, and we omit this basic ring in the notation for cohomology.) The degree of this element coincides with the (real) codimension of the stratum. The product of this element with the class $\psi$ taken to the complementary power is simply a rational number which, and this is the result of the geometrical approach suggested in [2], is closely related to the number of ramified coverings we are interested in. Before describing this connection let us give the rigorous statement of the Hurwitz problem.

Two ramified coverings $f_{1}: C_{1} \rightarrow S^{2}, f_{2}: C_{2} \rightarrow S^{2}$ of the 2-sphere are said to be isomorphic if there is a homeomorphism $h: C_{1} \rightarrow C_{2}$ such that $f_{1}=f_{2} \circ h$. Clearly, isomorphic ramified coverings have coinciding ramification points in the target sphere and coinciding ramification types over these points, as well as the degrees $n_{1}, n_{2}$ and genera $g_{1}, g_{2}$ of the covering surfaces, $n_{1}=n_{2}=n, g_{1}=g_{2}=g$. A ramification point in the target sphere is said to be nondegenerate, or simple, if it has $n-1$ geometrically distinct preimages one of which is a ramification point of multiplicity 2 , and the other $n-2$ points are smooth points of the covering. As it was mentioned above, we assign to each ramification point in the target the reduced partition $\alpha$ consisting of the multiplicities of its singular preimages decreased by one. The set of all reduced partitions $\alpha$ for a given ramified covering is called the ramification data. The Hurwitz number $h_{\alpha_{1}, \alpha_{2}, \ldots}$ is defined by the equation

$$
h_{\alpha_{1}, \alpha_{2}, \ldots}=\sum_{f} \frac{1}{|\operatorname{Aut}(f)|},
$$

where the summation is carried over all isomorphism classes of ramified coverings $f$ of the sphere of degree $n$ by a surface of genus $g$, with reduced partitions $\alpha_{1}, \alpha_{2}, \ldots$ over the degenerate ramification points, and $|\operatorname{Aut}(f)|$ denotes the number of elements in the automorphism group of such ramified covering (this group is always finite). Of course, the Hurwitz numbers depend also on $g$ and $n$, but we do not 
The Hurwitz problem consists in counting Hurwitz numbers, and it is related to the geometry of Hurwitz spaces by the following theorem, which has been explicitly formulated, for the case $g=0$, in [16] and which follows immediately from the results in [2].

Theorem 1.1. We have

$$
h_{\alpha_{1}, \alpha_{2}, \ldots}=\frac{\left|\operatorname{Aut}\left(\alpha_{1}, \alpha_{2}, \ldots\right)\right|}{n !}\left\langle\sigma_{\alpha_{1}, \alpha_{2}, \ldots}, \psi^{d}\right\rangle,
$$

where $\left|\operatorname{Aut}\left(\alpha_{1}, \alpha_{2}, \ldots\right)\right|$ denotes the order of the automorphism group of the set of reduced partitions, that is, the product of factorials of the numbers of pairwise coinciding partitions, and the angle brackets denote the multiplication in the cohomology of $P \overline{\mathcal{H}}_{g, n}$.

Theorem 1.1 reduces the Hurwitz problem to a problem about the cohomology of the Hurwitz spaces. In spite of the visible complication caused by replacing a groupcombinatorial problem with an algebro-geometric one, this geometric interpretation not only clarifies the known computational results (see [8], [6], [15], [2]), but also leads to new ones (see, e.g., [16]).

Generally speaking, the cohomology rings of moduli spaces of stable curves and closely related to them Hurwitz spaces are very complicated, even in the case of rational curves and functions $(g=0)$. Our experience shows, however, that all natural cohomology classes related to singularities (as well as multisingularities and multimultisingularities - these are exactly the classes playing the central role in the Hurwitz problem) can be expressed in terms of a small relatively simple set of "basic" (tautological, in a sense) classes. Our goal is to identify these basic classes, describe relations between them, and find expressions for strata in terms of these classes.

1.3. Description of main results. We start with describing results concerning Hurwitz numbers.

Theorem 1.2. For rational (i.e., related to the case $g=0$ ) Hurwitz numbers the following formulas are true:

$$
\begin{aligned}
& h_{2^{1}, 2^{1}}=\frac{3}{4}\left(27 n^{2}-137 n+180\right) \frac{(2 n-6) !}{(n-3) !} n^{n-6}, \\
& h_{2^{1}, 1^{2}}=3\left(3 n^{2}-15 n+20\right) \frac{(2 n-6) !}{(n-4) !} n^{n-6}, \\
& h_{1^{2}, 1^{2}}=2\left(2 n^{3}-16 n^{2}+43 n-40\right) \frac{(2 n-6) !}{(n-4) !} n^{n-6} .
\end{aligned}
$$

These formulas describe some multimultisingularities, that is, the case where the ramification is nondegenerate over several points in the target sphere. For the case of multisingularities, where the ramification is degenerate over a single point, the rational Hurwitz numbers are given by the well-known Hurwitz formula. The first formula in Theorem 1.2 was obtained by D. Zvonkine in [21], the other two, as far as we know, are new.

To prove these results, we deduce some relations in the cohomology of Hurwitz 
shall also make an extensive use of the class $\delta \in H^{2}\left(P \overline{\mathcal{H}}_{g, n}\right)$ represented by functions on singular curves. The class $\delta$ is very close to the pullback of the boundary class in $\overline{\mathcal{M}}_{g, n}$ with respect to the projection $\overline{\mathcal{H}}_{g, n} \rightarrow \overline{\mathcal{M}}_{g, n}$ (the difference of the two is the divisor of functions having a single pole on a rational irreducible component attached to other components at a single point). The value of the latter in the geometry of moduli spaces of stable curves is well-known. For example, the index of maximal selfintersection of this class is nothing but the Weil-Petersson volume of the moduli space (see [17]). The essential difference between Hurwitz spaces and moduli spaces of curves is arising due to existence of another natural class of 2-cohomology, namely, the class $\psi$.

Note that all the results presented in the paper are valid in the Chow ring of $P \overline{\mathcal{H}}_{g, n}$, not only in the cohomology. The only reason why we speak about cohomology is that the cohomological computations are sufficient for the expected computational applications.

As far as we know, the first relations for strata in the cohomology rings of Hurwitz spaces were obtained in [16]. They express the caustic and the Maxwell stratum in the spaces of rational functions $(g=0)$ in terms of the boundary stratum and the class $\psi$ :

$$
\begin{aligned}
\sigma_{2^{1}} & =6(n-1) \psi-3 \delta, \\
\sigma_{1^{2}} & =2(n-1)(n-6) \psi+4 \delta .
\end{aligned}
$$

We present below a new derivation of these formulas. The classes whose complex codimension is greater than one also are subject to numerous relations which are described in more detail in the main body of the paper, since their description requires additional definitions.

1.4. Degree. Theorem 1.1 reduces the process of calculating Hurwitz numbers to the computation of the intersection index of a stratum with the complementary power of the class $\psi$. Such class $\psi$ arises naturally on any orbit space of an action of the group $\mathbb{C}^{*}$; it behaves functorially under equivariant mappings of varieties endowed with actions of this group. That is why the intersection index of a subvariety with the complementary degree of this class deserves the name of degree of the subvariety.

Let $A$ be a complex variety and let the multiplicative group $\mathbb{C}^{*}$ of nonzero complex numbers act on $A$ without fixed points. Let $B$ be the orbit space of this action and suppose $B$ is compact. Denote by $\Psi=c_{1}(O(1)) \in H^{2}(B)$ the tautological class of this action. For an arbitrary class $\beta \in H^{*}(B)$, the degree $\operatorname{deg} \beta$ is the result of the pairing

$$
\operatorname{deg} \beta=\int_{B} \frac{\beta}{1-\Psi}=\int_{B} \beta\left(1+\Psi+\Psi^{2}+\Psi^{3}+\ldots\right) .
$$

In other words, the degree of a homogeneous class $\beta$ is the degree of its intersection with the class $\Psi$ taken to the power equal to the codimension of $\beta$.

Example 1.3. The weighted projective space $\mathbb{C} P_{w_{0}, \ldots, w_{n}}$ is the quotient space of the complement to the origin in the vector space $\mathbb{C}^{n+1}$ endowed with the following action of the group $\mathbb{C}^{*}$ : 
The integral exponents $w_{i}$ are the weights of the action. Each holomorphic linear representation of $\mathbb{C}^{*}$ has such a form. The degree of the weighted projective space $\mathbb{C} P_{w_{0}, \ldots, w_{n}}$ with the weights $w_{0}, \ldots, w_{n}$ is $\left(\prod w_{i}\right)^{-1}$.

The definitions immediately imply the functoriality of the degree.

Proposition 1.4. Suppose the group $\mathbb{C}^{*}$ acts without fixed points on complex varieties $A_{1}, A_{2}$ of the same dimension, and suppose the orbit spaces $B_{1}, B_{2}$ are compact. Then the degree of an equivariant mapping $f: A_{1} \rightarrow A_{2}$ is independent of $f$ and can be determined from the equality

$$
\operatorname{deg} B_{1}=\operatorname{deg} f \cdot \operatorname{deg} B_{2} \text {. }
$$

Theorem 1.1 is an immediate corollary of this Proposition applied to the mapping $L_{g, n}: \overline{\mathcal{H}}_{g, n} \rightarrow \mathbb{C}^{2 g+2 n-3}$ taking a meromorphic function to the unordered set of its critical values (recall that according to our agreement the sum of the critical values is zero).

1.5. Singularities, relative Chern classes, and universal polynomials. Equations (1.1) were obtained in [16] by computing the intersection indices of the classes $\sigma_{2^{1}}, \sigma_{1^{2}}$ with a basic set of classes of complementary dimension (i.e., of complex dimension one, that is, curves). However as the codimension of the strata grows this way of arguing becomes cumbersome: both the choice of a basic set of classes of complementary dimension and the computation of the intersection index with such a class are complicated problems, without known approaches to their effective solutions. We present a new approach to the search for relations between cohomology classes. This approach is based on Thom's theory of universal polynomials for singularities [20] extended to the case of multisingularities in [10]-[13]. Let us explain briefly the basics of this theory.

Let $F: M \rightarrow N$ be a generic holomorphic mapping of compact complex varieties (we assume, for definiteness, that the varieties have coinciding dimensions: although the theory works for arbitrary dimensions, we are going to apply it only in the case of coinciding dimensions). The total Chern class of $F$ is the ratio

$$
\begin{aligned}
c(F)= & \frac{c\left(F^{*} T N\right)}{c(T M)}=1+\left(c_{1}\left(F^{*}(T N)\right)-c_{1}(T M)\right)+\left(c_{2}\left(F^{*} T N\right)\right. \\
& \left.\quad-c_{1}\left(F^{*} T N\right) c_{1}(T M)-c_{2}(T M)+c_{1}^{2}(T M)\right)+\ldots \in H^{*}(M) ;
\end{aligned}
$$

here the function $c(\cdot)$ whose argument is a vector bundle denotes, as usual, the total Chern class of this bundle. The homogeneous components of the total Chern class are called the Chern classes of $F$ and denoted by $c_{i}(F) \in H^{2 i}(M)$ :

$$
\begin{aligned}
& c_{1}(F)=c_{1}\left(F^{*} T N\right)-c_{1}(T M), \\
& c_{2}(F)=c_{2}\left(F^{*} T N\right)-c_{1}\left(F^{*} T N\right) c_{1}(T M)-c_{2}(T M)+c_{1}^{2}(T M),
\end{aligned}
$$

and so on. A mapping $F$ has a singularity at a given point if its differential $d F$ is degenerate at this point. Singularities are split into various types. The closure of the set of points where $F$ has a singularity of a given type determines a cohomology class 
in the Chern classes of $F$, and the coefficients of the polynomial depend only on the type of the singularity, not on the varieties $M$ and $N$ or the mapping $f$.

The notion of both a generic mapping and a type of a singularity requires a more precise definition which can be found, e.g., in [13]. As the simplest example let us consider the singularity $A_{1}$ : a mapping has singularity $A_{1}$ at a given point if the rank of the differential of $F$ at this point is one less than the maximal possible, and the restriction of $F$ to the kernel of the differential is nondegenerate. For a general mapping the set of points where it has a singularity of type $A_{1}$ is a subvariety of complex codimension one. The cohomology class of this closure coincides with $c_{1}(F)=c_{1}\left(F^{*} T N\right)-c_{1}(T M)$, which can be easily shown by considering the pullback to $M$ of a nonzero differential form of the highest degree on $N$. Already in the simplest cases this expression for the class $A_{1}$ leads to nontrivial results. Suppose, for example, that the dimensions of both varieties is one, i.e., we deal with ramified coverings of degree, say, $n$ of a complex curve by a complex curve. Then we obtain the Riemann-Hurwitz formula for the number of ramification points of a generic ramified covering:

$$
c_{1}(F)=n\left(2-2 g_{N}\right)-\left(2-2 g_{M}\right)=2(n-1)-2\left(n g_{N}-g_{M}\right)
$$

where $g_{M}$ and $g_{N}$ are, respectively, the genus of the covering and of the covered surface.

A similar statement for multisingularities asserts that the closure in $N$ of the set of points such that $F$ has at their preimages singularities of prescribed types can be expressed as a universal polynomial in the pushforwards $F_{*}\left(\left(c_{1}(F)\right)^{k_{1}}\left(c_{2}(F)\right)^{k_{2}} \ldots\right) \in H^{*}(N)$ of monomials in Chern classes of $F$. We also extend this approach to the case of multimultisingularities, that is, subvarieties in moduli spaces of functions consisting of functions having prescribed sets of multisingularities.

We apply the methods of the theory of universal polynomials to the universal mapping over the Hurwitz space which we are going to define. Denote by $\overline{\mathcal{U}}_{g, n}$ the universal curve over $\overline{\mathcal{H}}_{g, n}$, i.e., a complex orbifold together with a mapping $\overline{\mathcal{U}}_{g, n} \rightarrow \overline{\mathcal{H}}_{g, n}$ whose fiber over a point $f \in \overline{\mathcal{H}}_{g, n}$ is the domain of definition of $f$ (or, more precisely, the quotient of this domain modulo the action of the automorphism group of the function $f$ ). One more variety is just the direct product $\mathbb{C} P^{1} \times \overline{\mathcal{H}}_{g, n}$, with a coordinate chosen in the first factor. The universal mapping over the Hurwitz space takes a point of the universal curve $\overline{\mathcal{U}}_{g, n}$ to the value of the corresponding function at this point. It is fibered over the Hurwitz space.

Remark 1.5. As it is well known, the universal curve over the moduli space of stable curves $\overline{\mathcal{M}}_{g, n}$ coincides with the moduli space $\overline{\mathcal{M}}_{g, n+1}$ of stable curves with $n+1$ marked points. Indeed, a point of a fiber of the universal curve can be treated as the $(n+1)$ st marked point along the fiber. A similar construction works for Hurwitz spaces as well.

The multiplicative group $\mathbb{C}^{*}$ of nonzero complex numbers acts on each of the three spaces $\overline{\mathcal{H}}_{g, n}, \overline{\mathcal{U}}_{g, n}$ and $\mathbb{C} P^{1} \times \overline{\mathcal{H}}_{g, n}$ by multiplying a function by a constant. On $\overline{\mathcal{U}}_{g, n}$ this action is fiberwise trivial, while on the fiber $\mathbb{C} P^{1}$ of the direct product the group $\mathbb{C}^{*}$ acts as multiplication by constants (this action is well defined since we have chosen a coordinate in the fiber). The universal mapping is equivariant 
with respect to the action. After restricting both the universal curve and the direct product to the complement to the set of fixed points of the action on $\overline{\mathcal{H}}_{g, n}$ and taking the quotients of the resulting spaces modulo the group actions, we arrive at the triangle of spaces and mappings which will be the main object of our study. Note that since the action of $\mathbb{C}^{*}$ on the fibers of the direct product $\mathbb{C} P^{1} \times \overline{\mathcal{H}}_{g, n}$ is nontrivial, its quotient does not coincide with the direct product $\mathbb{C} P^{1} \times P \overline{\mathcal{H}}_{g, n}$; instead, it is isomorphic to the projectivization of the vector bundle $\mathbb{C} \oplus \mathcal{O}(1)$ of rank two over $P \overline{\mathcal{H}}_{g, n}$.

Introduce the simplified notation: $B_{g, n}$ for the projectivized Hurwitz space $P \overline{\mathcal{H}}_{g, n}, \quad X_{g, n}$ for the quotient of the universal curve (which, in its own turn, is the universal curve over the projectivized Hurwitz space), $Y_{g, n}$ for the quotient of the direct product, $p_{g, n}$ (respectively, $q_{g, n}$ ) for the projection of $X_{g, n}$ (respectively, $\left.Y_{g, n}\right)$ to $B_{g, n}$ and $f_{g, n}$ for the quotient universal mapping $f_{g, n}: X_{g, n} \rightarrow Y_{g, n}$. In other words, we consider the commutative triangle

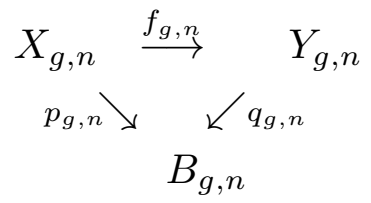

We start with showing that the relative Chern classes of the universal mapping can be expressed in terms of few "basic" classes in $H^{*}\left(X_{g, n}\right)$, and then describe the strata in the space $B_{g, n}$ in terms of the pushforwards of these basic classes. This proves to be realizable because the mapping $f_{g, n}$ has not so many types of singularities, and the types are independent of $g$ and $n$, which leads to universal formulas.

Note that since the Hurwitz space $B_{g, n}$ is the moduli space of stable meromorphic functions of degree $n$ on curves of genus $g$ and $X_{g, n}$ is the universal curve on $B_{g, n}$, any family of stable meromorphic functions of degree $n$ on curves of genus $g$ is induced from the projection $p_{g, n}: X_{g, n} \rightarrow B_{g, n}$. In particular, linear relations for cohomology classes valid in $X_{g, n}$ and $B_{g, n}$ remain valid in arbitrary family.

1.6. The structure of the paper. In $\S 2$ we analyze local singularities of the universal mapping and introduce the basic cohomology classes. In $\S 3$ we show that the relative Chern classes of the universal mapping indeed can be expressed in terms of the basic classes. Then we deduce formulas for some strata in $B_{g, n}$ in terms of the pushforwards of polynomials in basic classes. In $\S 4$ the degrees of the strata are computed.

The authors express their gratitude to B. Lass, D. Zagier and D. Zvonkine for useful discussions. The paper was completed during the second author's stay at the Max-Planck Institut für Mathematik, Bonn, Germany, in January-February 2004.

\section{$\S 2$. Singularities of FiBERED MAPPINGS WITH ONE-DIMENSIONAL FIBERS}

The relative Chern classes of the universal mapping $f_{g, n}$ are closely related to its singularities. Since the mapping $f_{g, n}$ is fibered over the base $B_{g, n}$ and the fibers are one-dimensional, these singularities are relatively simple. The classification of simple isolated singularities with one-dimensional fibers [5] includes singularities of types $A_{k}$ and $I_{k, l}$. The mappings $f_{g, n}$ acquire such singularities. However in 
cases $g=0$ and $n=2$ or $n=3$ ) nonisolated ones. The latter arise if a fiber which is the domain of the function contains an irreducible component where the function is constant. Note that such a function is not necessarily unstable: if the genus of the irreducible component is at least one, or it is rational but contains at least three nodes, then the function can be constant on such a component, and yet stable. Of course, our approach to the study of the geometry of Hurwitz spaces requires a complete investigation of nonisolated singularities, which we do not present here, whence we obtain only partial results. We plan to discuss nonisolated singularities in a separate paper.

2.1. Local singularities. Consider the commutative triangular diagram of spaces and holomorphic mappings of the form

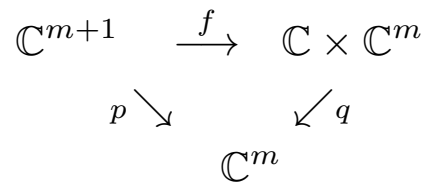

where $q$ is the projection of the direct product to the second factor. We assume that the fibers of $p$ are one-dimensional (although not necessarily smooth). Restricting $f$ to the fibers of $p$ we obtain a family of functions on (noncompact) curves. The natural "right" equivalence of such diagrams admits arbitrary coordinate changes in the base $\mathbb{C}^{m}$, as well as fiberwise coordinate changes in $\mathbb{C}^{m+1}$ fibered over the chosen coordinate transformation in the base. We extend this equivalence group allowing for multiplicating a function by a constant depending holomorphically on the point of the base. This extension does not affect the classification of singularities, but leads to a richer theory of characteristic classes.

Since the mapping $q$ is a projection, and $p$ is the composition of $f$ and $q$, the singularities of the diagram are totally determined by those of $f$. A point $x \in \mathbb{C}^{m+1}$ is a local singularity of $f$ if the rank of the differential of $f$ at $x$ is less than $m+1$. Let us make the following two assumptions:

- the mapping $f$ has only generic singularities, i.e., the complement to the set of such singularities in the space of jets of diagrams is a closed nowhere dense subset;

- the projection $p: \mathbb{C}^{m+1} \rightarrow \mathbb{C}^{m}$ has no singularities that are more complicated than the Morse folding $(x, y) \mapsto x y$.

For the universal mapping we are interested in, the second assumption is satisfied, while the first one is not: the restriction of the function to a fiber can prove to be constant on some irreducible component of the fiber. As a result, the formulas we obtain are valid "up to some classes supported on the subvariety of nonisolated singularities". In the case of rational functions the codimension of the subvariety of nonisolated singularities is 3 , which allows us to obtain exact formulas for the strata up to codimension two.

Under the above assumptions the mapping $f$ can have singularities of the following types:

(1) the type $A_{k}, \quad k=1,2, \ldots$, is realized at those smooth points of the fibers of $p$, where the restriction of $f$ to the fiber can be reduced to the form $x \mapsto x^{k+1}$ in an appropriate local coordinate $x$ along the fiber;

(2) the type $I_{k, l}, \quad k \geq l \geq 1$, is realized at those singular points of the fibers of $p$, where the restriction of $f$ to the plane with the local coordinates $x, y$, such that $p$ 
At those points one can choose coordinates on the base $\mathbb{C}^{m}$ such that $f$ can be represented as the direct sum of the identity mapping and miniversal unfoldings which, for the cases $A_{k}$ and $I_{k, l}$, look as follows:

(1) $A_{k}:\left(x, b_{2}, \ldots, b_{k}\right) \mapsto\left(x^{k+1}+b_{2} x^{k-1}+\cdots+b_{k} x, b_{2}, \ldots, b_{k}\right)$;

(2) $I_{k, l}:\left(x, y, a_{1}, \ldots, a_{k-1}, b_{1}, \ldots, b_{l-1}\right) \mapsto\left(x^{k}+a_{1} x^{k-1}+\cdots+a_{k-1} x+y^{l}+\right.$ $\left.b_{1} y^{l-1}+\cdots+b_{l-1} y, x y, a_{1}, \ldots, a_{k-1}, b_{1}, \ldots, b_{l-1}\right)$.

We interpret these unfoldings as globally given polynomial mappings of complex vector spaces of coinciding dimensions. In the case $A_{k}$ (respectively, $I_{k, l}$ ) such an unfolding is the universal mapping of the universal curve over the moduli space of rational functions with a single pole of order $k+1$ (respectively, with two poles, of orders $k$ and $l$ ) [14]. More precisely, this is not the moduli space, but some finite covering of the moduli space, of degree $k+1$ in the case $A_{k}$ and of degree $k l$ in the case $I_{k, l}$. Therefore, in the above coordinates the multiplication of a function by a constant produces the multiplication of a rational function of the type $A_{k}$ (respectively, $I_{k, l}$ ) by $\lambda^{k+1} \in \mathbb{C}^{*}$ (respectively, $\lambda^{k l}$ ) which, after the substitution $x \mapsto \lambda x$, defines the action of the group $\mathbb{C}^{*}$ on the unfoldings according to the following formulas:

(1) for the singularity $A_{k}$

$$
\lambda:\left(x, b_{2}, \ldots, b_{k}\right) \mapsto\left(\lambda x, \lambda^{2} b_{2}, \ldots, \lambda^{k} b_{k}\right), \quad \lambda \in \mathbb{C}^{*}
$$

(2) for the singularity $I_{k, l}$

$$
\begin{aligned}
\lambda:\left(x, y, a_{1}, \ldots, a_{k-1}, b_{1}, \ldots, b_{l-1}\right) & \\
& \mapsto\left(\lambda^{l} x, \lambda^{k} y, \lambda^{l} a_{1}, \ldots, \lambda^{l(k-1)} a_{k-1}, \lambda^{k} b_{1}, \ldots, \lambda^{k(l-1)} b_{l-1}\right), \quad \lambda \in \mathbb{C}^{*} .
\end{aligned}
$$

2.2. Characteristic classes of singularities. Suppose that diagram (2.1) has a singularity of the type $A_{k}$ at the origin. Without loss of generality one can suppose that $k=m+1$, and $f$ is exactly the above miniversal unfolding of the singularity $A_{k}$. The space $\mathbb{C}^{k}$ in the upper left corner of diagram (2.1) contains subvarieties corresponding to all singularities $A_{i}$ for $i \leq k$. These subvarieties are invariant with respect to the action of the group $\mathbb{C}^{*}$, therefore, to the singularity $A_{i}$ a subvariety $\left[A_{i}\right]$ in the weighted projective space $\mathbb{C} P^{(k)}=\mathbb{C} P_{1,2, \ldots, k}$ obtained by factorization of the punctured space $\mathbb{C}^{k} \backslash\{0\}$ modulo the action of the group $\mathbb{C}^{*}$ described above is assigned, whence a cohomology class $\left[A_{i}\right] \in H^{2 i}\left(\mathbb{C} P^{(k)}\right)$. Denote by $\Sigma \in H^{2}\left(\mathbb{C} P^{(k)}\right)$ the characteristic class of the singularity $A_{1}$, i.e., the first Chern class of $f$, and by $\Psi \in H^{2}\left(\mathbb{C} P^{(k)}\right)$ the characteristic class of the action of $\mathbb{C}^{*}$.

Theorem 2.1. The characteristic classes of the singularities $\left[A_{i}\right]$ in the unfoldings of the singularities $A_{k}$ can be expressed in terms of $\Sigma$ and $\Psi$ according to the formula

$$
\left[A_{i}\right]=P_{i}(\Sigma, \Psi)=\Sigma(2 \Sigma-\Psi)(3 \Sigma-2 \Psi) \ldots(i \Sigma-(i-1) \Psi) .
$$

In particular, the expressions for these classes in terms of $\Psi$ and $\Sigma$ are independent of the order $k$ of the perturbed singularity $A_{k}$.

Proof. Denote by $t \in H^{2}\left(\mathbb{C} P^{(k)}\right)$ the cohomology class such that $\Psi=(k+1) t$. Then $t$ is a generator of the cohomology ring $H^{*}\left(\mathbb{C} P^{(k)}\right)$. Because of quasihomogenity, we have $\Sigma=k t$. The expression for the class $\left[A_{i}\right]$ in terms of $t$ and $k$ must have the form 
for some constant $c_{i}$. Here the Pohgammer symbol $(k)_{i}$ denotes the following polynomial in $k$ of degree $i$ :

$$
(k)_{i}=k(k-1)(k-2) \ldots(k-i+1) .
$$

Indeed, Thom's theorem together with Corollary 3.4 below imply that the class $\left[A_{i}\right]$ admits some universal representation as a homogeneous polynomial in $\Psi$ and $\Sigma$. Substituting into this polynomial the expressions for $\Psi$ and $\Sigma$ in terms of $t$ and $k$, we conclude that the class $\left[A_{i}\right]$ is proportional to $t^{i}$ and the proportionality coefficient is a polynomial in $k$ of degree $i$ vanishing for all $k<i$. For $i=k$ we have $\left[A_{i}\right]=i$ ! $t^{i}$ which yields $c_{i}=1$. Now the expression for $A[i]$ in terms of $\Psi$ and $\Sigma$ results from the substitution $t=\Psi-\Sigma, k=\Sigma /(\Psi-\Sigma)$. The theorem is proved.

The miniversal unfolding of the singularity $I_{k, l}$ contains both the strata corresponding to the singularities $A_{i}$ for $i<k+l$ and the strata corresponding to the singularities of the type $I_{i, j}$ for $i \leq k$ and $j \leq l$. These strata are also invariant with respect to the action of the group $\mathbb{C}^{*}$. Taking the quotient modulo this action, we obtain the weighted projective space $\mathbb{C} P^{(k, l)}=\mathbb{C} P_{l, l, 2 l, \ldots,(k-1) l, k, k, 2 k, \ldots,(l-1) k}$, and all the cohomology classes considered below belong to the cohomology of this space.

Singularities of the type $I_{i, j}$ have support on the subvariety $\Delta$ of singular points of the fibers of the projection $p$. In the coordinates chosen above this subvariety is given by the equations $x=y=0$. Let us add to the classes $\Psi, \Sigma \in H^{2}\left(\mathbb{C} P^{(k, l)}\right)$ the class $\Delta \in H^{4}\left(\mathbb{C} P^{(k, l)}\right)$. We also consider the normal bundle to $\Delta$ and denote by $N$ its first Chern class. The class $\Delta$ can be interpreted as the second Chern class of this normal bundle, and the classes $N$ and $\Delta$ can be considered as (commuting) operators acting on $\Delta$. Even less formally, one may treat $N$ as a second cohomology class $N \in H^{2}\left(\mathbb{C} P^{(k, l)}\right)$ (which makes sense only when intersected with $\Delta$ ). We are interested in expressions for singularities of the type $A_{i}$ in the unfoldings of singularities of the type $I_{k, l}$ in terms of the classes $\Sigma, \Psi, \Delta, N$.

Theorem 2.2. There are expressions for the classes $\left[A_{i}\right]$ in the unfoldings of singularities $I_{k, l}$ as quasihomogeneous polynomials of degree $i$ in the variables $\Sigma, \Psi, \Delta, N$, such that:

(i) these polynomials are universal, i.e., they depend on $i$ but not on $k$ and $l$;

(ii) the polynomial corresponding to the class $\left[A_{i}\right]$ can be represented as a sum of two polynomials, the first summand coinciding with the polynomial $P_{i}(\Sigma, \Psi)$ considered above, and the second summand having the support on the stratum $\Delta$ of double points;

(iii) each polynomial supported on $\Delta$ can be represented as a polynomial in $\Delta, N$ and $\Psi$.

The first two statements follow from Thom's theorem and Corollary 3.4 below, while the third one is a corollary of the obvious relation $\Sigma \Delta=\Psi \Delta$.

Denote the polynomial corresponding to the class $\left[A_{i}\right]$ by $R_{i}$. It has the form $R_{i}=P_{i}+Q_{i-2} \Delta$, where the polynomial $P_{i}$ is already known and $Q_{i-2}=$ $Q_{i-2}(\Delta, N, \Psi)$ is a quasihomogeneous polynomial of degree $i-2$. We did not manage to find an explicit formula for these polynomials, however, they can be computed using the indeterminate coefficients method. Let us show how this method works 
Similarly to the above situation, each element in $H^{*}\left(\mathbb{C} P^{(k, l)}\right)$ can be expressed as a polynomial in $t=\frac{\Psi}{k l}$. Knowing the weights of quasihomogenity, we conclude, in particular, that

$$
\Psi=\Sigma=k l t, \quad N=(k+l) t, \quad \Delta=k l t^{2} .
$$

Let us find, using this representation, the coefficients of the polynomials $Q_{0}$ and $Q_{1}$. We know that $Q_{0}=a$ for some constant $a$ and that the stratum $A_{2}$ in the unfolding of the singularity $I_{1,1}$ is empty. For $k=l=1$ we have $\Psi=\Sigma=t$, $\Delta=t^{2}$, whence

$$
R_{2}=\Sigma(2 \Sigma-\Psi)+a \Delta=t^{2}+a t^{2}=0 .
$$

Therefore, $a=-1$, i.e.,

$$
R_{2}=\Sigma(2 \Sigma-\Psi)-\Delta .
$$

Similarly, $Q_{1}=a N+b \Psi$ for some constants $a$ and $b$. Using the fact that the stratum $A_{3}$ is empty in the unfoldings of each of the singularities $I_{1,1}$ and $I_{2,1}$, we obtain the following system of linear equations:

$$
\begin{aligned}
1+2 a+b & =0 \\
8+6 a+4 b & =0
\end{aligned}
$$

(the homogeneity allowed us do divide both equations by $t^{3}$ ), whence $a=2, b=-5$, i.e.,

$$
Q_{1}=2 N-5 \Psi
$$

Similar calculations yield

$$
\begin{aligned}
Q_{2}= & -\left(6 N^{2}-15 N \Psi+15 \Psi^{2}-8 \Delta\right), \\
Q_{3}= & 24 N^{3}-62 N^{2} \Psi+63 N \Psi^{2}-35 \Psi^{3}-60 N \Delta+84 \Psi \Delta, \\
Q_{4}=- & \left(120 N^{4}-322 N^{3} \Psi+343 N^{2} \Psi^{2}-196 N \Psi^{3}+70 \Psi^{4}\right. \\
& \left.\quad-432 N^{2} \Delta+812 N \Psi \Delta-469 \Psi^{2} \Delta+180 \Delta^{2}\right) .
\end{aligned}
$$

Note that the number of indeterminate coefficients in the expansion of $A_{i}$ is $\left[i^{2} / 4\right]$, where the square brackets denote the integral part of a number, and this value exactly coincides with the number of singularities of the type $I_{k, l}$ whose miniversal unfoldings do not contain the stratum $A_{i}$ because of the dimensional reasons.

2.3. Residual polynomials for multisingularities. Fix a partition $\alpha=$ $1^{m_{1}} 2^{m_{2}} \ldots$ (where only finitely many exponents are nonzero). Consider the set of points $y$ in the space $\mathbb{C} \times \mathbb{C}^{m}$ in the upper right corner of the commutative triangle (2.1) possessing the following properties:

- the fiber $p^{-1}(q(y))$ of the projection $p$ is nonsingular;

- the restriction of $f$ to this fiber has, among the preimages of $y$, exactly $m_{1}$ instances of the singularity $A_{1}$, exactly $m_{2}$ instances of the singularity $A_{2}$ and so on (in addition to singularities of the prescribed types, the point $y$ may have also nonsingular preimages, whose number is determined by the degree of $f$ ).

Denote the class of the closure of this set times the order $m_{1} ! m_{2} ! \ldots$ of the 
(The multiplication by the order of the automorphism group allows one to get rid of fractions in the further explicit calculations of the classes.)

Were the pushforwards $f_{*} R_{k}$ of the subvarieties $R_{k}=A_{k}$ intersecting transversally, the classes $A_{1^{m_{1}} 2^{m_{2}} \ldots}(f)$ would be described by the coefficients of $\frac{t_{1}^{m_{1}}}{m_{1} !} \frac{t_{2}^{m_{2}}}{m_{2} !} \ldots$ in the exponent of the generating function $f_{*}\left(R_{1} t_{1}+R_{2} t_{2}+\ldots\right)$. (The factorials $m_{i}$ ! in the denominators reflect the possibility to permute the preimages with the same singularity types.) The nontransversality of the intersections makes it necessary to introduce some correction terms, the residual polynomials [10].

Consider the exponential generating function

$$
\mathcal{A}(f)=\sum\left[A_{\left.1^{m_{1} 2^{m_{2}} \ldots}(f)\right]} \frac{t_{1}^{m_{1}}}{m_{1} !} \frac{t_{2}^{m_{2}}}{m_{2} !} \ldots\right.
$$

where the summation is carried over all partitions $\alpha=1^{m_{1}} 2^{m_{2}} \ldots$, including the empty partition, and the square brackets denote the cohomology classes Poincaré dual to the corresponding subvarieties. Let us introduce also the exponential generating function in the infinite set of variables $t_{1}, t_{2}, \ldots$, with formal coefficients $R_{\alpha}$, where $\alpha$ takes values in the set of all nonzero partitions:

$$
\mathcal{R}\left(t_{1}, t_{2}, \ldots\right)=\sum R_{1^{m_{1} 2^{m_{2}}} \ldots} \frac{t_{1}^{m_{1}}}{m_{1} !} \frac{t_{2}^{m_{2}}}{m_{2} !} \ldots
$$

Proposition 2.3. There are weighted homogeneous polynomials $R_{\alpha}$ in the classes $\Sigma, \Psi, \Delta, N$ such that after substituting them for the coefficients in the generating series $\mathcal{R}$ the cohomology classes of the strata $A_{1^{m_{1}} 2^{m_{2}} \ldots}(f)$ coincide with the coefficients of the monomials $\frac{t_{1}^{m_{1}}}{m_{1} !} \frac{t_{2}^{m_{2}}}{m_{2} !} \ldots$ in the exponent $\exp \left(f_{*} \mathcal{R}\right)$ :

$$
\mathcal{A}(f)=\exp \left(f_{*} \mathcal{R}\left(t_{1}, t_{2}, \ldots\right)\right)
$$

Here the symbol $f_{*}$ denotes the Gysin homomorphism, that is, the direct image homomorphism in the cohomology of the quotient spaces modulo the actions of the group $\mathbb{C}^{*}$. This mapping is a homomorphism of the additive, not of the multiplicative structure. The polynomials $R_{\alpha}$ introduced in this way are called the residual polynomials for multisingularities. Of course, there is nothing strange in the fact that the polynomials $R_{k^{1}}$ coincide with the polynomials $R_{k}$ introduced in Sec. 2.2 (indeed, the corresponding points have only one singular preimage).

Proof. The most essential part of the Proposition states that the correction term

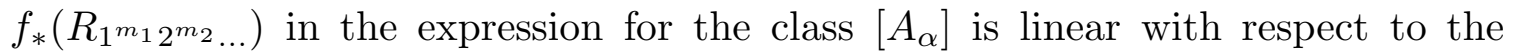
pushforwarded classes. The main observation allowing one to prove this statement consists in the fact that the universal expression for the multisingularities classes remains valid even in the case of disconnected underlying curves of meromorphic functions.

Let us apply this remark to a generic family of curves

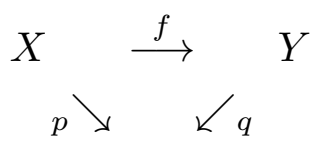


Consider the mapping $f^{(d)}: X^{(d)} \rightarrow Y$, where $X^{(d)}$ denotes the space fibered over $B$, which is a disjoint union of $d$ copies of $X, X^{(d)}=X_{1} \sqcup \cdots \sqcup X_{d}, X_{i}=X$, and $f^{(d)}$ is the mapping whose restriction to the $i$ th copy $X_{i}$ coincides with $f$. To be more precise, we take for the restriction of $f^{(d)}$ to $X_{i}$ a slightly perturbed copy of $f$, which is necessary for making $f^{(d)}$ general.

Since the expression for classes of multisingularities is universal, it can be applied to each of the mappings $f^{(d)}$ constructed above. It is easy to see that

$$
\mathcal{A}\left(f^{(d)}\right)=(\mathcal{A}(f))^{d} .
$$

Indeed, each partition $\alpha$ corresponding to a multisingularity of $f^{(d)}$ splits into $d$ subpartitions each corresponding to a multisingularity of one of the components of $f^{(d)}$. The combinatorial coefficients of these partitions are exactly the ones arising in the process of raising to the power $d$. We conclude that

$$
\log \mathcal{A}\left(f^{(d)}\right)=d \log \mathcal{A}(f) .
$$

On the other hand, it is obvious that the pushforward of any monomial in the basic classes under the mapping $f^{(d)}$ coincides with the $d$-fold pushforward of this monomial under $f$. Therefore, none of the coefficients in the generating function $\log \mathcal{A}(f)$ contains a product of the pushforwards of basic classes (the degree of the contribution of such coefficients would be greater than $d$ ), whence all the coefficients are linear. The Proposition is proved.

To compute the residual polynomials, we must know how the homomorphism $f_{*}$ acts on the cohomology classes we are interested in. The target of $f$ also is a weighted homogeneous space, and its cohomology ring is generated by a hyperplane class, which we also denote by $t$. Hence $f_{*}$ has the form $f_{*}: t^{i} \mapsto c t^{i}$ for some constant $c$. This constant can be nothing but the constant $f_{*} 1$, that is, the degree of $f$. If $f$ is a miniversal deformation of the singularity $A_{k}$, then its degree equals $k+$ 1.

Let us start with computing the polynomials $R_{\alpha}$ for standard unfoldings of the singularities of the type $A_{k}$ (i.e., in the presence of singularities of the type $A$ solely). These polynomials depend only on the classes $\Sigma$ and $\Psi$. They can be computed in the following way.

After substituting $\Sigma=k t, \Psi=(k+1) t$ into the generating function $\mathcal{R}$ we must obtain

$$
\begin{gathered}
\exp \left((k+1) \mathcal{R}\left(t_{1}, t_{2}, \ldots\right)\right)=1+(k+1)_{2} \frac{t_{1}}{1 !} t+\left((k+1)_{3} \frac{t_{2}}{1 !}+(k+1)_{4} \frac{t_{1}^{2}}{2 !}\right) t^{2} \\
+\left((k+1)_{4} \frac{t_{3}}{1 !}+(k+1)_{5} \frac{t_{1}}{1 !} \frac{t_{2}}{1 !}+(k+1)_{6} \frac{t_{1}^{3}}{3 !}\right) t^{3}+\ldots
\end{gathered}
$$

Indeed, the coefficient of the monomial $\frac{t_{1}^{i_{1}}}{i_{1} !} \frac{t_{2}^{i_{2}}}{i_{2} !} \ldots$ in the exponent must have the form $t^{i_{1}+2 i_{2}+3 i_{3}+\ldots}$ with a coefficient that is a polynomial in $k$ vanishing for all $k$ up to the codimension of the corresponding stratum, $k=-1,0,1, \ldots, 2 i_{1}+3 i_{2}+\cdots-2$.

Note that the right-hand side of Eq. (2.3) can be conveniently rewritten in the form $^{1}$

$$
\exp \left((k+1) \mathcal{R}\left(t_{1}, t_{2}, \ldots\right)\right)=\left.e^{\tau_{1} \tau+\tau_{2} \tau^{2}+\tau_{3} \tau^{3}+\ldots} s^{k+1}\right|_{s=1} .
$$


Here $s$ is an additional variable commuting with all $t_{i}$ and $t$, and we use the notation

$$
\tau=t \frac{d}{d s}, \quad \tau_{i}=t_{i} \frac{d}{d s} .
$$

Now it is easy to compute the residual polynomials $R_{\alpha}$ by taking the logarithm of the right-hand side of Eq. (2.3), dividing it by $(k+1)$ and making the substitution $t=\Psi-\Sigma, \quad k=\Sigma /(\Psi-\Sigma)$. For small codimensions we obtain

$$
\begin{aligned}
R_{1^{2}} & =-2 \Sigma(3 \Sigma-\Psi), \\
R_{1^{1} 2^{1}} & =-6 \Sigma(2 \Sigma-\Psi)^{2}, \\
R_{1^{3}} & =8 \Sigma\left(15 \Sigma^{2}-13 \Sigma \Psi+3 \Psi^{2}\right), \\
R_{1^{1} 3^{1}} & =-4 \Sigma(5 \Sigma-3 \Psi)(3 \Sigma-2 \Psi)(2 \Sigma-\Psi), \\
R_{2^{2}} & =-3 \Sigma(2 \Sigma-\Psi)\left(20 \Sigma^{2}-25 \Sigma \Psi+8 \Psi^{2}\right), \\
R_{1^{2} 2^{1}} & =24 \Sigma(2 \Sigma-\Psi)\left(15 \Sigma^{2}-17 \Sigma \Psi+5 \Psi^{2}\right), \\
R_{1^{4}} & =-48 \Sigma\left(105 \Sigma^{3}-160 \Sigma^{2} \Psi+84 \Sigma \Psi^{2}-15 \Psi^{3}\right) .
\end{aligned}
$$

The $\Delta$-part of the residual polynomials in the unfoldings of the singularities $I_{k, l}$ can be computed recursively using the indeterminate coefficients method, as above.

Let us compute, for example, the polynomial $R_{1^{2}}$. Due to the weighted homogeneity it has the form

$$
R_{1^{2}}=2 \Sigma \Psi-6 \Sigma^{2}+a \Delta
$$

for some constant $a$ (we know already the $\Delta$-free part), and the stratum $A_{1^{2}}$ can be represented as the unknown coefficient of $t_{1}^{2} / 2$ ! in $\exp \left(f_{*} \mathcal{R}\right)$ :

$$
\left[A_{1^{2}}\right]=\left(f_{*} R_{1^{1}}\right)^{2}+f_{*} R_{1^{2}}=\left(f_{*} \Sigma\right)^{2}+f_{*}\left(2 \Sigma \Psi-6 \Sigma^{2}+a \Delta\right) .
$$

The degree of the unfolding of the singularity $I_{k, l}$ is $k+l$. Substituting the expressions for the classes $\Psi, \Delta, \Sigma$ in terms of the class $t$ in the unfolding of the singularity $I_{1,1}$ and making use of the fact that the class $A_{1^{2}}$ is empty in this unfolding, we obtain the linear equation

$$
2^{2}+4-12+2 a=0
$$

whence $a=2$, i.e.,

$$
R_{1^{2}}=2\left(\Sigma \Psi-3 \Sigma^{2}+\Delta\right)
$$

Similar calculations for residual polynomials in higher codimensions lead to the following answers:

$$
\begin{aligned}
R_{1^{1} 2^{1}}= & -6 \Sigma(2 \Sigma-\Psi)^{2}+6 \Delta\left(3 \Psi-N_{1}\right), \\
R_{1^{3}}= & 8 \Sigma\left(15 \Sigma^{2}-13 \Sigma \Psi+3 \Psi^{2}\right)-8 \Delta(10 \Psi-3 N), \\
R_{1^{1} 3^{1}}=- & 4 \Sigma(5 \Sigma-3 \Psi)(3 \Sigma-2 \Psi)(2 \Sigma-\Psi) \\
& +4 \Delta\left(20 \Psi^{2}-17 N \Psi+6 N^{2}-8 \Delta\right), \\
R_{2^{2}}=- & 3 \Sigma(2 \Sigma-\Psi)\left(20 \Sigma^{2}-25 \Sigma \Psi+8 \Psi^{2}\right) \\
& +3 \Delta\left(25 \Psi^{2}-21 N \Psi+8 N^{2}-12 \Delta\right), \\
R_{1^{2} 2^{1}}= & 24 \Sigma(2 \Sigma-\Psi)\left(15 \Sigma^{2}-17 \Sigma \Psi+5 \Psi^{2}\right) \\
& \quad-24 \Delta\left(20 \Psi^{2}-15 N \Psi+5 N^{2}-7 \Delta\right), \\
R_{1^{4}}=- & 48 \Sigma\left(105 \Sigma^{3}-160 \Sigma^{2} \Psi+84 \Sigma \Psi^{2}-15 \Psi^{3}\right) \\
& +48 \Delta\left(70 \Psi^{2}-48 N \Psi+15 N^{2}-21 \Delta\right) .
\end{aligned}
$$


2.4. Residual polynomials for multimultisingularities. Now we are interested in subvarieties of the space in the lower corner of the triangle (2.1) consisting of points $b$ such that the restriction of $f$ to the fiber $p^{-1}(b)$ has several degenerate multisingularities, with prescribed reduced partitions $\alpha_{1}^{m_{1}}, \ldots, \alpha_{c}^{m_{c}}$ (as well as the necessary number of nondegenerate singularities). The exponents in the notation indicate the numbers of corresponding partitions. Denote by $A_{\alpha_{1}^{m_{1}}, \ldots, \alpha_{c}^{m_{c}}}(f) \subset \mathbb{C}^{m}$ the closure of the set of such points multiplied, similarly to the multisingularity case, by the product of the orders of the automorphism groups of the partitions $\alpha_{i}$ and by the order of the automorphism group of the set of the partitions $\alpha_{i}$.

Our calculations show that the following statement, to which we did not find a formal proof yet, holds.

Conjecture 2.4. Under the assumptions stated in the beginning of $\S 2$, each class of multisingularities can be given as a universal polynomial combination of the images of monomials in the basic classes under the Gysin homomorphism $p_{*}$.

Similarly to the multisingularities case, the very existence of universal polynomials implies that they must have a rather special form and be represented in terms of residual polynomials in the basic classes.

Consider the infinite set of variables $s_{1^{2}}, s_{2^{1}}, s_{1^{2}, 1^{2}}, s_{1^{2}, 2^{1}}, s_{2^{1}, 2^{1}}, \ldots$ indexed by sets of partitions $\alpha$ (say, lexicographically ordered). Define a multiplication on this set by setting the product of two variables equal to the sum of variables of the same kind, whose indices are all sets of partitions that can be obtained by uniting some pairwise distinct partitions in the index of the second variable with some pairwise distinct partitions in the index of the first variable. For example,

$$
s_{\alpha, \beta} s_{\gamma, \delta}=s_{\alpha, \beta, \gamma, \delta}+s_{\alpha \gamma, \beta, \delta}+s_{\alpha, \beta \gamma, \delta}+s_{\alpha \delta, \beta, \gamma}+s_{\alpha, \beta \delta, \gamma}+s_{\alpha \gamma, \beta \delta}+s_{\alpha \delta, \beta \gamma} .
$$

In particular, each polynomial in the variables $s_{\alpha_{1}, \alpha_{2}, \ldots}$ coincides with a linear polynomial.

Let us introduce the generating function for multimultisingularities by the formula

$$
\mathfrak{A}\left(s_{1^{2}}, s_{2^{1}}, \ldots\right)=\sum \frac{\left[A_{\left.\alpha_{1}^{m_{1}}, \ldots, \alpha_{c}^{m_{c}}\right]}\right.}{\prod \operatorname{Aut}\left(\alpha_{i}\right)} \frac{s_{\alpha_{1}^{m_{1}}, \ldots, \alpha_{c}^{m_{c}}}}{m_{1} ! \ldots m_{c} !} .
$$

Consider also the exponential generating function with the formal coefficients $T_{\alpha_{1}^{m_{1}}, \ldots, \alpha_{c}^{m_{c}}}$, indexed by the multipartitions:

$$
\mathfrak{R}\left(s_{1^{2}}, s_{2^{1}}, \ldots\right)=\sum \frac{T_{\alpha_{1}^{m_{1}}, \ldots, \alpha_{c}^{m_{c}}}}{\prod \operatorname{Aut}\left(\alpha_{i}\right)} \frac{s_{\alpha_{1}^{m_{1}}, \ldots, \alpha_{c}^{m_{c}}}}{m_{1} ! \ldots m_{c} !} .
$$

Proposition 2.5. Under Conjecture 2.4, if we take for $T_{\alpha}$ the polynomials representing the classes $A_{\alpha}$, then there are weighted homogeneous polynomials $R_{\alpha_{1}^{m_{1}}, \ldots, \alpha_{c}^{m_{c}}}$, for $c \geq 2$, in the classes $\Sigma, \Psi, \Delta, N$ such that after substituting their pushforwards $f_{*} R_{\alpha_{1}, \ldots, \alpha_{c}}$ for the coefficients $T_{\alpha_{1}^{m_{1}}, \ldots, \alpha_{c}^{m_{c}}}$ in the above generating function $\mathfrak{R}$ the cohomology classes of the strata $A_{\alpha_{1}^{m_{1}}, \ldots, \alpha_{c}^{m_{c}}}$ coincide with the coefficients of $\frac{s_{\alpha_{1}^{m_{1}}, \ldots, \alpha_{c}^{m_{c}}}}{m_{1} ! \ldots m_{c} !}$ in $\exp \left(q_{*} \mathfrak{R}\right)$, where the exponent is taken with respect to the multiplication of the variables s described above.

This statement is proved exactly in the same way as Proposition 2.3 by consid- 
As above, the polynomials $R_{\alpha_{1}, \ldots, \alpha_{c}}$ can be computed inductively by means of the indeterminate coefficients method. Calculations make use of the fact that the restriction of $p$ to $\Sigma$ is a proper mapping, and all the cohomology classes we are interested in are supported on $\Sigma$ and hence the homomorphism $p_{*}$ expressed in terms of the basic class $t$ is nothing but multiplication by the degree of the restriction $\left.p\right|_{\Sigma}$. The latter degree coincides with the number of the critical points of $f$ on a general fiber and is equal to $k+1$ for the singularity $A_{k}$ and $k+l$ for the singularity $I_{k, l}$. Here are the first nontrivial results of the calculations:

$$
\begin{aligned}
& R_{2^{1}, 2^{1}}=-2 \Sigma(2 \Sigma-\Psi)(5 \Sigma-3 \Psi)+\Delta(14 \Psi-5 N) \\
& R_{2^{1}, 1^{2}}=6 \Sigma(2 \Sigma-\Psi)(5 \Sigma-2 \Psi)-6 \Delta(7 \Psi-2 N), \\
& R_{1^{2}, 1^{2}}=-6 \Sigma\left(30 \Sigma^{2}-21 \Psi \Sigma+4 \Psi^{2}\right)+2 \Delta(57 \Psi-14 N) .
\end{aligned}
$$

\section{$\S 3$. Relative Chern classes of the universal mapping}

In this section we show that the relative Chern classes of the universal mapping indeed do belong to the subring in $H^{*}\left(X_{g, n}\right)$ generated by the basic classes. This means that the classes $\left[A_{k}\right]$ as well as the residual polynomials for multisingularities and multimultisingularities also belong to this subring. The main tool in the calculation of relative Chern classes of $f_{g, n}$ is the Grothendieck-Riemann-Roch theorem. This theorem implies also nontrivial relations for the Gysin pushforwards of polynomials in the basic classes. We start with recalling the theorem in the specific situation where we are going to use it. Our approach follows that of [3], [18].

3.1. Grothendieck-Riemann-Roch theorem. The Grothendieck-RiemannRoch (GRR) theorem relates the Chern character of a coherent sheaf over a variety $M$ with the Chern character of the direct image of this sheaf under a mapping $P: M \rightarrow A$. Suppose that both varieties $M$ and $A$ are compact and smooth.

In one of its forms (maybe, the most useful one) the GRR formula looks like

$$
\operatorname{ch}\left(P_{!} \beta\right)=P_{*}\left(\operatorname{ch}(\beta) \operatorname{td}(P)^{-1}\right) .
$$

Here $\beta$ is a coherent sheaf on $M$, ch denotes the Chern character, and td denotes the Todd class of the morphism $P$.

Let us give the definitions of the objects participating in the GRR formula.

3.1.1. Chern character. The Chern character of the sheaf of sections of a vector bundle, or simply the Chern character of a vector bundle, is defined as the sum of the exponents of its Chern roots, that is, the formal roots of its Chern polynomial,

$$
\operatorname{ch}(E)=\exp \left(e_{1}\right)+\cdots+\exp \left(e_{r}\right)
$$

where $r$ denotes the rank of the vector bundle $E$. In particular, for a line bundle $E$ we have $\operatorname{ch}(E)=\exp \left(c_{1}(E)\right)$.

Being a symmetric function in the Chern roots, the Chern character of a bundle can be expressed in terms of its characteristic classes:

$$
\begin{aligned}
\operatorname{ch}(E)=r & +c_{1}+\frac{1}{2}\left(c_{1}^{2}-2 c_{2}\right)+\frac{1}{6}\left(c_{1}^{3}-3 c_{1} c_{2}+3 c_{3}\right) \\
& +\frac{1}{24}\left(c_{1}^{4}-4 c_{1}^{2} c_{2}+4 c_{1} c_{3}+2 c_{2}^{2}-4 c_{4}\right) \\
& +\frac{1}{}\left(c_{1}^{5}-5 c_{1}^{3} c_{0}+5 c_{1} c_{2}^{2}+5 c_{1}^{2} c_{2}-5 c_{0} c_{2}-5 c_{1} c_{1}+5 c_{5}\right)+
\end{aligned}
$$


where $c_{i}$ denotes the $i$ th symmetric function of the Chern roots.

Because of the properties of Chern roots, an exact sequence of vector bundles

$$
0 \longrightarrow E^{\prime} \longrightarrow E \longrightarrow E^{\prime \prime} \longrightarrow 0
$$

leads to the Whitney formula

$$
\operatorname{ch}(E)=\operatorname{ch}\left(E^{\prime}\right)+\operatorname{ch}\left(E^{\prime \prime}\right)
$$

The Whitney formula allows one to extend the notion of Chern character to arbitrary coherent sheaves. A coherent sheaf on $M$ is either a sheaf of sections of a vector bundle or, more generally, a sheaf that can be inserted as the last nontrivial term into an exact sequence of sheaves all whose terms but the last one are sheaves of sections of vector bundles (such an exact sequence is called the resolvent for the given sheaf). The Chern character of a coherent sheaf is defined as the alternated sum of the Chern characters of the other terms in a resolvent.

For the structure sheaf of a subvariety $\Sigma \subset M$ of codimension one in $M$ given by an embedding $i: \Sigma \hookrightarrow M$, the resolvent looks like follows:

$$
0 \longrightarrow N_{\Sigma}^{\vee} \longrightarrow \mathcal{O}_{M} \longrightarrow i_{*}\left(\mathcal{O}_{\Sigma}\right) \longrightarrow 0
$$

where $N_{\Sigma}$ is a line bundle over $M$ such that $\Sigma$ coincides with the set of zeros of its section. In a tubular neighborhood of $\Sigma$ the bundle $N_{\Sigma}$ coincides with the natural extension of the normal bundle to $\Sigma$ in $M$. The Whitney formula implies the identity

$$
\begin{aligned}
\operatorname{ch}\left(i_{*}\left(\mathcal{O}_{\Sigma}\right)\right) & =\operatorname{ch}\left(\mathcal{O}_{M}\right)-\operatorname{ch}\left(N_{\Sigma}^{\vee}\right)=1-\exp (-\Sigma) \\
& =\frac{(1-\exp (-\Sigma))}{\Sigma} \Sigma=\left(\frac{\Sigma}{1-\exp (-\Sigma)}\right)^{-1} \Sigma,
\end{aligned}
$$

and the operator of intersection with the first Chern class of the normal bundle can be replaced with $\Sigma$, since, by assumption, the variety $\Sigma$ coincides with the zero locus of a holomorphic section of the normal bundle.

For a subvariety $Z$ of codimension two the resolvent proves to be more complicated, namely, it has the form

$$
0 \longrightarrow \Lambda^{2} N_{Z}^{\vee} \longrightarrow N_{Z}^{\vee} \longrightarrow \mathcal{O}_{M} \longrightarrow i_{*}\left(\mathcal{O}_{Z}\right) \longrightarrow 0
$$

where $\Lambda^{2}$ denotes the skew-symmetric square of a vector bundle. This sequence, called the Koszul resolvent, is written under the assumption that $Z$ is the zero locus of a globally defined section of some rank two vector bundle $N_{Z}$ over $M$. We shall see that the final expression for $\operatorname{ch}\left(i_{*}\left(\mathcal{O}_{Z}\right)\right)$ is uniquely determined by the restriction of the bundle $N_{Z}$ to $Z$, which coincides with the normal bundle to $Z$. Moreover, one can show (see [3]) that the expression thus obtained remains valid without assuming that the normal bundle to $Z$ can be extended to the entire variety $M$. The Whitney formula implies 
The rank of the virtual vector bundle $N_{Z}^{\vee}$ is 2 , while its total Chern class is $c\left(N_{Z}^{\vee}\right)=1-N_{1}+N_{2}$, where $N_{i}=c_{i}\left(N_{Z}\right), i=1,2$. Therefore, its Chern character has the form

$$
\begin{aligned}
\operatorname{ch}\left(N_{Z}^{\vee}\right)=2 & -N_{1}+\frac{1}{2}\left(N_{1}^{2}-2 N_{2}\right)+\frac{1}{6}\left(3 N_{1} N_{2}-N_{1}^{3}\right)+\frac{1}{24}\left(N_{1}^{4}-4 N_{1}^{2} N_{2}+2 N_{2}^{2}\right) \\
& -\frac{1}{120}\left(N_{1}^{5}-5 N_{1}^{3} N_{2}+5 N_{1} N_{2}^{2}\right)+\ldots
\end{aligned}
$$

The vector bundle $\Lambda^{2} N_{Z}^{\vee}$ is a line bundle and

$$
c\left(\Lambda^{2} N_{Z}^{\vee}\right)=1-N_{1}
$$

whence

$$
\operatorname{ch}\left(\Lambda^{2} N_{Z}^{\vee}\right)=\exp \left(-N_{1}\right)
$$

Combining these results we obtain

$$
\begin{aligned}
\operatorname{ch}\left(i_{*} \mathcal{O}_{Z}\right) & =N_{2}-\frac{1}{2} N_{1} N_{2}+\ldots \\
& =\left(1-\frac{1}{2} N_{1}+\frac{1}{12}\left(N_{1}^{2}-N_{2}\right)-\frac{1}{24}\left(N_{1}^{3}-N_{1} N_{2}\right)+\ldots\right) Z
\end{aligned}
$$

since both $Z$ and $N_{2}$ are identified with the class of the zero section of the normal bundle $N_{Z}$.

3.1.2. Todd class. We start with the definition of the Todd class of a vector bundle, and then define the Todd class of a mapping. The Todd class of a vector bundle $E$ over $M$ is another symmetric function of its Chern roots,

$$
\begin{aligned}
\operatorname{td}(E)= & \frac{e_{1}}{1-\exp \left(-e_{1}\right)} \cdots \frac{e_{r}}{1-\exp \left(-e_{r}\right)}=1+\frac{1}{2} c_{1}+\frac{1}{12}\left(c_{1}^{2}+c_{2}\right) \\
& +\frac{1}{24} c_{1} c_{2}+\frac{1}{720}\left(-c_{1}^{4}+4 c_{1}^{2} c_{2}+3 c_{2}^{2}+c_{1} c_{3}-c_{4}\right)+\ldots
\end{aligned}
$$

For the above exact sequence of vector bundles we have

$$
\operatorname{td}(E)=\operatorname{td}\left(E^{\prime}\right) \operatorname{td}\left(E^{\prime \prime}\right)
$$

For any vector bundle $E$ of rank $r$ we have

$$
\sum_{i=0}^{r}(-1)^{i} \operatorname{ch}\left(\Lambda^{i} E^{\vee}\right)=c_{r}(E) \operatorname{td}(E)^{-1}
$$

The Todd class of a variety $M$ is the Todd class of its tangent bundle $T M$. The Todd class of a proper mapping $F: M \rightarrow N$ is the Todd class of the difference $F^{*} T N-T M$, i.e.,

$$
\operatorname{td}(F)=\operatorname{td}\left(F^{*} T N-T M\right)=\frac{\operatorname{td} F^{*} T N}{\operatorname{td} T M}
$$


3.1.3. Direct image of a coherent sheaf. The higher direct image $P_{!}(E)$ of a coherent sheaf $E$ on $M$ with respect to a mapping $P: M \rightarrow A$ is the most complicated object among those defined in the present section. Methods of its computing depend on the difference between the dimensions of the varieties $A$ and $M$. In particular, if the dimensions of the varieties coincide, then the image $P_{!}(E)$ coincides with the pushforward $P_{*}(E)$. Recall that by definition the pushforward $P_{*} E$ of a sheaf $E$ over $M$ with respect to a mapping $P: M \rightarrow A$ is the sheaf whose group of sections over a sufficiently small open set $U \subset A$ coincides with the restriction of $E$ to $P^{-1}(U)$. If $\operatorname{dim} M=\operatorname{dim} A+1$ (this is exactly the situation where we are going to apply the GRR theorem), then

$$
P_{!}(E)=R^{0}\left(P_{*}(E)\right)-R^{1}\left(P_{*}(E)\right),
$$

where $R^{0}\left(P_{*}(E)\right)=P_{*}(E)$ is the pushforward of $E$ and $R^{1}\left(P_{*}(E)\right)$ is the first higher Grothendieck direct image which can be computed by means of the Serre duality:

$$
R^{1}\left(P_{*}(E)\right)=\left(R^{0}\left(P_{*}\left(E^{\vee} \otimes \omega\right)\right)\right)^{\vee}=\left(P_{*}\left(E^{\vee} \otimes \omega\right)\right)^{\vee} ;
$$

here $\omega$ is the relative dualizing sheaf.

3.2. Relative Chern classes of the universal mapping. Now let us return to the commutative triangle (1.2) of spaces and mappings. From now on we shall use simplified notation omitting the indices $g, n$ : we denote the space $X_{g, n}$ by $X$, the mapping $f_{g, n}$ by $f$, and so on. Introduce in $X$ (recall that $X$ is the universal curve over the projectivized Hurwitz space) the following cohomology classes, which we call basic classes. Denote by $\omega$ the relative dualizing sheaf of the mapping $p: X \rightarrow B$, i.e., the unique holomorphic line bundle over $X$ whose fiber over a smooth point of a fiber of $p$ coincides with the cotangent line to this fiber. The first Chern class $c_{1}(\omega)$ is an element of the second cohomology group $H^{2}(X)$. Let $\Pi$ be the divisor of poles of the universal mapping $f$, that is, the locus of points in $X$ where the restriction of $f$ to the fiber has a pole. Recall that since each of the spaces $X, Y$ and $B$ is a space of orbits under an action of the group $\mathbb{C}^{*}$, a cohomology class $c_{1}(\mathcal{O}(1)) \in H^{2}$ is distinguished on each of them; we denote these classes by $\Psi_{X}$, $\Psi_{Y}$ and $\psi$, respectively. Introduce also the notation $\Sigma=c_{1}(\omega)+\Psi_{X}+2 \Pi$ for the first relative Chern class of $f$.

In addition to subvarieties and classes of codimension one we shall also make use of the class $\Delta \subset X$ represented by the set of singular points of the fibers of $p$. The rank of the normal bundle to $\Delta$ in $X$ is 2 . Locally this normal bundle admits a natural splitting into the direct sum of two line bundles.

Indeed, each point in $\Delta$ is a point of transversal intersection of the two branches of the fiber at this point, and that are the tangent lines to these branches that produce the splitting of the normal bundle. (In the case of rational curves, $g=0$, this splitting extends to a global splitting since a double point cuts the rational curve into two connected components; we shall not make use of this construction, however). Denote by $N$ the first Chern class of the normal bundle to $\Delta \subset X$. This Chern class can be treated as an operator that can be applied to $\Delta$. Its degrees $N^{i}$ are operators of the same form. The second Chern class of the normal bundle can be identified with the class $\Delta$ itself.

Lemma 3.1. The basic classes satisfy the following relations (in the cohomology $\left.H^{*}(X)\right)$ : 
Proof. The identity $\Pi \Delta=0$ is a corollary of the fact that the poles of a function in the Hurwitz space are smooth points of the underlying curve, whence the subvarieties $\Delta$ and $\Pi$ are disjoint. Similarly, the identity $\Sigma \Pi=0$ expresses the fact that all the poles of a function are simple, and there is no ramification over infinity.

The dualizing sheaf $\omega$ is almost trivial over $\Delta$ (its pullback to the two-fold covering of $\Delta$ is trivial), and it is isomorphic to the conormal line bundle to the hypersurface $\Pi$ at points of this hypersurface. These properties imply the identities $c_{1}(\omega) \Delta=0$ and $\Pi^{2}=-c_{1}(\omega) \Pi$. Substituting $c_{1}(\omega)=\Sigma-\Psi_{X}-2 \Pi$ into these equations we obtain, respectively,

$$
\begin{aligned}
0 & =\left(\Sigma-\Psi_{X}-2 \Pi\right) \Delta=\Sigma \Delta-\Psi_{X} \Delta, \\
\Pi^{2} & =-\left(\Sigma-\Psi_{X}-2 \Pi\right) \Pi=\Psi_{X} \Pi+2 \Pi^{2},
\end{aligned}
$$

and the identities $\Sigma \Delta=\Psi_{X} \Delta$ and $\Pi^{2}=-\Psi_{X} \Pi$ follow. Note that the latter can also be obtained by pulling back to $X$ the identity $\Pi_{Y}^{2}=-\Psi_{Y} \Pi_{Y}$, which takes place on $Y$ (see Sec. 3.3 below).

Corollary 3.2. Each polynomial in the basic classes can be represented in the form

$$
P_{1}\left(\Psi_{X}\right) \Pi+P_{2}\left(\Psi_{X}, \Sigma\right)+P_{3}\left(\Psi_{X}, N, \Delta\right) \Delta
$$

where $P_{1}, P_{2}$, and $P_{3}$ are some polynomials.

The main goal of the present section consists in the proof of the following statement, from which we shall deduce some relations for the pushforwards of the basic classes.

Theorem 3.3. The total relative Chern class of $f$ is

$$
c(f)=\left(1+\Psi_{X}\right)\left(\frac{1}{1-\Sigma+\Psi_{X}}-\frac{\Delta}{1+N+\Delta}\right) .
$$

The theorem immediately implies

Corollary 3.4. If the mapping $f$ can have singularities only of types $A_{k}$ or $I_{k, l}$, when restricted to the fibers of $p$, then the residual polynomials $R_{\alpha_{1}, \ldots, \alpha_{c}}$ for multisingularities belong to the subring in $H^{*}(X)$ generated by the basic classes $\Sigma, \Psi_{X}, \Delta, N$.

Since $p$ is the composition of the mappings $f$ and $q, p=f \circ q$, its total Chern class is the ratio of the pullback of the total relative Chern class of $q$ and the total relative Chern class of $f$ :

$$
c(f)=\frac{f^{*} c(q)}{c(p)} .
$$

Therefore, in order to compute this class it suffices to compute the classes $c(p)$, $c(q)$, as well as the pullback of the latter class to $X$. This is precisely what we are going to do now.

3.2.1. The class $c(q)$ and its pullback $f^{*} c(q)$. The total space $Y$ of the bundle $q$ 
by $\mathcal{T}$ the tautological line bundle over $Y$ and set $\Pi_{Y}=c_{1}\left(\mathcal{T}^{\vee}\right)=-c_{1}(\mathcal{T})$. This class satisfies the relation $c_{2}\left(q^{*}(E) / \mathcal{T}\right)=0$, i.e.,

$$
\Pi_{Y}^{2}+\Pi_{Y} \Psi_{Y}=0
$$

where $\Psi_{Y}=q^{*}(\psi)=c_{1}(\mathcal{O}(1))$ is the characteristic class of the action of the group $\mathbb{C}^{*}$. The resulting relation describes the cohomology ring of $Y$ as an algebra over $H^{*}(B)$, and the action of the ring $H^{*}(B)$ is determined by the mapping $q^{*}$.

The lines $\mathbb{C} \oplus\{0\}$ and $\{0\} \oplus \mathcal{O}(1)$ in the fibers of $E$ correspond to the points " 0 " and " $\infty$ " in the fibers of $q$. These points form hypersurfaces with the Poincaré dual classes

$$
c_{1}(\operatorname{Hom}(\mathcal{T}, \mathcal{O}(1)))=\Pi_{Y}+\Psi_{Y}, \quad c_{1}(\operatorname{Hom}(\mathcal{T}, \mathbb{C}))=\Pi_{Y}
$$

respectively, hence the class $\Pi_{Y}$ can be interpreted as the "dual class to the set of infinities in $Y$ ", and Eq. (3.4) expresses the fact that the set of zeros and the set of infinities are disjoint.

The total Chern class of $q$ is inverse to the total Chern class of the relative tangent bundle $\operatorname{ker}\left(q_{*}\right)$. This bundle is given by the isomorphism $\operatorname{ker}\left(q_{*}\right) \simeq$ $\operatorname{Hom}\left(\mathcal{T}, q^{*}(E) / \mathcal{T}\right)$. Therefore,

$$
c(q)=\frac{1}{c\left(\operatorname{Hom}\left(\mathcal{T}, q^{*}(E) / \mathcal{T}\right)\right)}=\frac{1}{1+\Psi_{Y}+2 \Pi_{Y}} .
$$

Applying $f^{*}$ we obtain $f^{*}\left(\Psi_{Y}\right)=\Psi_{X}$ and $f^{*}\left(\Pi_{Y}\right)=\Pi$ and, finally,

$$
f^{*}(c(q))=\frac{1}{1+\Psi_{X}+2 \Pi} .
$$

3.2.2. The total Chern class of $p$. The computation of the total Chern class of the mapping $p$ is slightly more complicated. It can be computed using the exact sequence

$$
0 \longrightarrow p^{*} T^{\vee} B \stackrel{p^{\vee}}{\longrightarrow} T^{\vee} X \longrightarrow \omega \longrightarrow \mathcal{O}_{\Delta} \longrightarrow 0,
$$

where, recall, $\omega$ denotes the relative dualizing sheaf of $p$. The subvariety $\Delta \subset X$ is exactly the set of those points where the corank of $p^{*}$ fails to be one and is 2 . The structure sheaf $\mathcal{O}_{\Delta}$ of the subvariety $\Delta$ measures the difference between the relative dualizing sheaf $\omega$ and the sheaf of relative differentials.

Applying to the above exact sequence the Whitney formula, we obtain

$$
c\left(p^{\vee}\right)=1-c_{1}(p)+c_{2}(p)-\ldots=c\left(p^{*} T^{\vee} B-T^{\vee} X\right)=\frac{c\left(\mathcal{O}_{\Delta}\right)}{c(\omega)}=\frac{c\left(\mathcal{O}_{\Delta}\right)}{1+c_{1}(\omega)} .
$$

To compute the Chern class $c\left(\mathcal{O}_{\Delta}\right)$ let us use the Koszul projective resolvent (3.2). Applying the Whitney formula once again we have

$$
c\left(\mathcal{O}_{\Delta}\right)=\frac{1-N}{1-N+\Delta}=1-\frac{\Delta}{1-N+\Delta} .
$$

This formula can be deduced as well by applying the GRR formula to the embedding $\Delta \hookrightarrow X$. Substituting it in the expression for $c\left(p^{\vee}\right)$, we obtain

$$
c(p)=\frac{1}{u}\left(1-\frac{\Delta}{1}\right) .
$$


Now we can compute the total Chern class of $f$. Applying the relations in Lemma 3.1, we have

$$
\begin{aligned}
c(f) & =\frac{c(p)}{f^{*}(c(q))}=\frac{1+\Psi_{X}+2 \Pi}{1-c_{1}(\omega)}\left(1-\frac{\Delta}{1+N+\Delta}\right) \\
& =\frac{1+\Psi_{X}+2 \Pi}{1-\Sigma+\Psi_{X}+2 \Pi}-\frac{1+\Psi_{X}}{1+N+\Delta} \Delta \\
& =\left(\frac{1+\Psi_{X}}{1-\Sigma+\Psi_{X}}-\frac{1+\Psi_{X}}{1+N+\Delta} \Delta\right)+\left(\frac{1+\Psi_{X}+2 \Pi}{1-\Sigma+\Psi_{X}+2 \Pi}-\frac{1+\Psi_{X}}{1-\Sigma+\Psi_{X}}\right) .
\end{aligned}
$$

The first summand coincides with the right-hand side of Eq. (3.3); after reducing the second summand to the common denominator its numerator becomes

$$
\left(1+\Psi_{X}+2 \Pi\right)\left(1-\Sigma+\Psi_{X}\right)-\left(1+\Psi_{X}\right)\left(1-\Sigma+\Psi_{X}+2 \Pi\right)=-2 \Pi \Sigma=0 .
$$

Theorem 3.3 is proved.

3.3. Pushforward homomorphisms. In order to be able to apply the universal formula to classes of multimultisingularities, we must have a description of the pushforward homomorphisms $f_{*}, p_{*}, q_{*}$. The following theorem states that the homomorphism $p_{*}$ uniquely determines the two others.

Theorem 3.5. For any set of cohomology classes $h_{1}, \ldots, h_{s}$ on $X$ satisfying the property $h_{i} \Pi=0, \quad i=1, \ldots, s$, we have

$$
q_{*}\left(f_{*}\left(h_{1}\right) \ldots f_{*}\left(h_{s}\right)\right)=\psi^{s-1} p_{*}\left(h_{1}\right) \ldots p_{*}\left(h_{s}\right) .
$$

The assumption $h_{i} \Pi=0$ means geometrically that the class $h_{i}$ admits a representation by a cycle nonintersecting the pole divisor $\Pi$. It is satisfied for all the residual polynomials of multisingularities.

Proof. Since $q$ is the projectivization of a vector bundle, the direct image $f_{*}(h)$ of an arbitrary class $h \in H^{*}(X)$ can be expressed in the form

$$
f_{*}(h)=q^{*}(a)+q^{*}(b) \Pi_{Y}
$$

for some classes $a$ and $b$ on $B$. The class $b$ can be computed by applying $q_{*}$ to both sides of this identity: the identity $q_{*} \Pi_{Y}=1$ together with the projection formula yields

$$
b=q_{*}\left(f_{*}(h)\right)=p_{*}(h) .
$$

The class a can be computed in a similar way: multiplying both sides of the above equation by $\Pi_{Y}+\Psi_{Y}$, we obtain

$$
f_{*}\left(\left(\Psi_{X}+\Pi\right) h\right)=q^{*}(a)\left(\Psi_{Y}+\Pi_{Y}\right)+q^{*}(b)\left(\Psi_{Y}+\Pi_{Y}\right) \Pi_{Y}=q^{*}(\psi a)+q^{*}(a) \Pi_{Y},
$$

and applying $q_{*}$ we get $a=p_{*}\left(\left(\Psi_{X}+\Pi\right) h\right)$. If the class $h$ satisfies the assumption of the theorem, $h \Pi=0$, then the answer can be simplified: 
Therefore, under the assumptions of the theorem we have

$$
f_{*}\left(h_{1}\right) \ldots f_{*}\left(h_{s}\right)=\left(\Psi_{Y}+\Pi_{Y}\right)^{s} \prod_{i=1}^{s} q^{*}\left(p_{*}\left(h_{i}\right)\right)=\left(\Psi_{Y}^{s}+\Psi_{Y}^{s-1} \Pi_{Y}\right) \prod_{i=1}^{s} q^{*}\left(p_{*}\left(h_{i}\right)\right) .
$$

Applying $q_{*}$ and the projection formula, we obtain the desired equation.

Hence to complete the calculation of the cohomology classes of the strata in the Hurwitz space $P \overline{\mathcal{H}}_{g, n}$ we must know how to compute the pushforward $p_{*}(R)$ of any residual polynomial expressed in the form

$$
R=P_{1}\left(\Psi_{X}, \Sigma\right)+P_{2}\left(\Psi_{X}, N, \Delta\right) \Delta .
$$

Let us set

$$
\xi_{k}=p_{*}\left(\Sigma^{k+1}\right), \quad \delta_{k, \ell}=p_{*}\left(N^{k} \Delta^{\ell+1}\right) .
$$

There are no universal relations between the classes $\xi_{k}$ and $\delta_{k, \ell}$ that are valid for all values of $g$ and $n$. Of course, for any given $g$ and $n$ the dimension of the cohomology space $H^{*}\left(P \overline{\mathcal{H}}_{g, n}\right)$ is finite, hence there are such relations. Some of them are discussed in Sec.3.4 below.

3.4. Relations on cohomology classes in $B$. Let us apply the GRR formula to the projection $p: X \rightarrow B$ and to the relative dualizing sheaf $\omega$. We proceed similarly to [18], where the same thing has been done for the projection of the universal curve to the moduli space of curves. We have

$$
\operatorname{ch}\left(p_{!} \omega\right)=p_{*}\left(\operatorname{ch}(\omega) \operatorname{td}(p)^{-1}\right) .
$$

The sheaf $p_{!} \omega=R^{0} p_{*} \omega-R^{1} p_{*} \omega$ on the left-hand side can be expressed in terms of the Hodge bundle $\Lambda=\Lambda_{g, n}$ over $B=P \overline{\mathcal{H}}_{g, n}$ (recall that the fiber of the Hodge bundle over a point $(C ; f)$ is the space of holomorphic 1 -forms over $C$; hence the rank of the bundle is $g$ ): $R^{0} p_{*} \omega=p_{*} \omega=\Lambda$ and, by the Serre duality, $R^{1} p_{*} \omega=$ $p_{*} \mathbb{C}=\mathbb{C}$. The classes on the right-hand side of the formula were computed above. Using the relation $c_{1}(\omega) \Delta=0$, we obtain

$$
\begin{aligned}
\operatorname{ch}(\omega) \operatorname{td}^{-1}(p) & =\operatorname{ch}(\omega) \operatorname{td}\left(\omega^{\vee}-\mathcal{O}_{\Delta}^{\vee}\right)=e^{c_{1}(\omega)} \frac{-c_{1}(\omega)}{1-e^{c_{1}(\omega)}} \operatorname{td}\left(-\mathcal{O}_{\Delta}^{\vee}\right) \\
& =\frac{c_{1}(\omega)}{1-e^{-c_{1}(\omega)}}+\operatorname{td}\left(-\mathcal{O}_{\Delta}^{\vee}\right)
\end{aligned}
$$

where the Todd class of the (virtual) sheaf $-\mathcal{O}_{\Delta}^{\vee}$ is totally determined by its Chern class

$$
c\left(-\mathcal{O}_{\Delta}^{\vee}\right)=\frac{1+N+\Delta}{1+N}=1+\frac{\Delta}{1+N} .
$$

Substituting this result into the GRR formula, we obtain

$$
\begin{gathered}
(g-1)+\operatorname{ch}_{1}(\Lambda)+\operatorname{ch}_{2}(\Lambda)+\operatorname{ch}_{3}(\Lambda)+\operatorname{ch}_{4}(\Lambda)+\operatorname{ch}_{5}(\Lambda)+\ldots \\
=\frac{1}{2} p_{*}\left(c_{1}(\omega)\right)+\frac{1}{12} p_{*}\left(c_{1}^{2}(\omega)+\Delta\right)-\frac{1}{720} p_{*}\left(c_{1}^{4}(\omega)+\left(N^{2}-3 \Delta\right) \Delta\right) \\
\quad+\frac{1}{}
\end{gathered}
$$


This relation shows that the homogeneous components of the Chern character (whence, the Chern classes) of the Hodge bundle can be expressed in terms of the pushforwards of the basic classes.

Note that there are no terms of positive even degrees on the right-hand side of Eq. (3.6). This means that the even part of the character $\operatorname{ch}(\Lambda)$ is trivial, i.e., $c\left(\Lambda+\Lambda^{\vee}\right)=1$. If $g=0$, then the Hodge bundle itself is trivial, whence the lefthand side of Eq. (3.6) vanishes at positive degrees. This property implies nontrivial identities for the pushforwards of the basic classes:

$$
p_{*}\left(c_{1}^{2}(\omega)+\Delta\right)=0, \quad p_{*}\left(c_{1}^{4}(\omega)+\left(N^{2}-3 \Delta\right) \Delta\right)=0, \quad \ldots
$$

\section{$\S 4$. Applying universal polynomials to} STUDYING THE STRATIFICATION OF HURWITZ SPACES

The knowledge of the residual polynomials for singularities, multisingularities, and multimultisingularities allows one to compute the cohomology classes of the strata in the Hurwitz spaces. Complete formulas of this kind must take into account the contribution of nonisolated singularities as well, which we did not manage to compute yet. That is why we present the universal expressions only for strata in the case of rational functions, and only for small codimensions.

4.1. Universal expressions for strata. Below we present the expressions for strata of codimensions 1 and 2 modulo classes supported on strata of nonisolated singularities in terms of direct images of the basic classes obtained on the base of the residual classes computed above (we use the notation $\xi_{k}=p_{*}\left(\Sigma^{k+1}\right), \quad \xi_{0}=$ $\left.2 n-2+2 g, \quad \delta_{k, l}=p_{*}\left(N^{k} \Delta^{l+1}\right)\right)$ :

$$
\begin{aligned}
& \sigma_{2^{1}}=-\psi \xi_{0}+2 \xi_{1}-\delta_{0,0} \\
& \sigma_{1^{2}}=\frac{1}{2} \psi \xi_{0}\left(\xi_{0}+2\right)-3 \xi_{1}+\delta_{0,0}, \\
& \sigma_{3^{1}}=2 \xi_{0} \psi^{2}-7 \xi_{1} \psi-5 \delta_{0,0} \psi+6 \xi_{2}+2 \delta_{1,0}, \\
& \sigma_{1^{1} 2^{1}}=-\xi_{0}\left(\xi_{0}+6\right) \psi^{2}+2\left(\xi_{0}+12\right) \xi_{1} \psi+\left(18-\xi_{0}\right) \delta_{0,0} \psi-24 \xi_{2}-6 \delta_{1,0}, \\
& \sigma_{1^{3}}=\frac{1}{6} \xi_{0}\left(\xi_{0}^{2}+6 \xi_{0}+24\right) \psi^{2}-\frac{1}{3}\left(9 \xi_{0}+52\right) \xi_{1} \psi \\
& +\frac{1}{3}\left(3 \xi_{0}-40\right) \delta_{0,0} \psi+20 \xi_{2}+4 \delta_{1,0}, \\
& \sigma_{2^{1}, 2^{1}}=\frac{1}{2}\left(\xi_{0}-6\right) \xi_{0} \psi^{2}-\left(2 \xi_{0}-11\right) \xi_{1} \psi+\frac{1}{2} \delta_{0,0}^{2}+2 \xi_{1}^{2}-10 \xi_{2} \\
& +\left(\xi_{0}+7\right) \delta_{0,0} \psi-2 \xi_{1} \delta_{0,0}-\frac{5}{2} \delta_{1,0}, \\
& \sigma_{2^{1}, 1^{2}}=-\frac{1}{2} \xi_{0}\left(\xi_{0}^{2}-2 \xi_{0}-12\right) \psi^{2}+\left(\xi_{0}^{2}+\xi_{0}-27\right) \xi_{1} \psi-\delta_{0,0}^{2}-6 \xi_{1}^{2}+30 \xi_{2} \\
& -\frac{1}{2}\left(\xi_{0}^{2}+42\right) \delta_{0,0} \psi+5 \xi_{1} \delta_{0,0}+6 \delta_{1,0}, \\
& \sigma_{1^{2}, 1^{2}}=\frac{1}{8}\left(\xi_{0}-4\right) \xi_{0}\left(\xi_{0}^{2}+4 \xi_{0}+6\right) \psi^{2}-\frac{3}{4}\left(2 \xi_{0}^{2}-4 \xi_{0}-21\right) \xi_{1} \psi+\frac{1}{2} \delta_{0,0}^{2} \\
& +\frac{9}{+} \xi^{2}-\frac{45}{-} \xi_{0}+\frac{1}{-}\left(2 \xi^{2}-4 \xi_{0}+57\right) \delta_{0} / 2-3 \xi_{1} \delta_{0}-\frac{7}{-} \delta_{1}
\end{aligned}
$$


Now let us give the expressions for the same strata in the case $g=0$ simplified with the help of the identities

$$
\xi_{0}=2 n-2, \quad \xi_{1}=4(n-1) \psi-\delta_{0,0}
$$

deduced in Sec. 3.4 (note that in the case $g=0$ the codimension of the locus of nonisolated singularities in $B$ is 3 , and hence they do not contribute to the above equations):

$$
\begin{aligned}
\sigma_{2^{1}}= & 6(n-1) \psi-3 \delta_{0,0} \\
\sigma_{1^{2}}= & 2(n-6)(n-1) \psi+4 \delta_{0,0}, \\
\sigma_{3^{1}}= & -24(n-1) \psi^{2}+2 \delta_{0,0} \psi+6 \xi_{2}+2 \delta_{1,0}, \\
\sigma_{1^{1} 2^{1}}= & 12(n-1)(n+6) \psi^{2}-6 n \delta_{0,0} \psi-24 \xi_{2}-6 \delta_{1,0}, \\
\sigma_{1^{3}}= & \frac{4}{3}(n-1)\left(n^{2}-17 n-30\right) \psi^{2}+4(2 n-1) \delta_{0,0} \psi+20 \xi_{2}+4 \delta_{1,0}, \\
\sigma_{2^{1}, 2^{1}}= & 2(n-1)(9 n+10) \psi^{2}-2(9 n-7) \delta_{0,0} \psi+\frac{9}{2} \delta_{0,0}^{2}-10 \xi_{2}-\frac{5}{2} \delta_{1,0}, \\
\sigma_{2^{1}, 1^{2}}= & 12(n-9)(n-1) n \psi^{2}-6\left(n^{2}-13 n+11\right) \delta_{0,0} \psi-12 \delta_{0,0}^{2}+30 \xi_{2}+6 \delta_{1,0}, \\
\sigma_{1^{2}, 1^{2}}= & (n-1)\left(2 n^{3}-30 n^{2}+145 n-60\right) \psi^{2}+\frac{1}{2}\left(16 n^{2}-144 n+125\right) \delta_{0,0} \psi \\
& \quad+8 \delta_{0,0}^{2}-\frac{45}{2} \xi_{2}-\frac{7}{2} \delta_{1,0} .
\end{aligned}
$$

4.2. Degrees of strata in genus zero. In the case $g=0$ the compactified Hurwitz space $P \overline{\mathcal{H}}_{0, n}$ is fibered over the compactified moduli space $\overline{\mathcal{M}}_{0, n}$ of rational curves with marked points. The fiber of this bundle is the projective space $P E$, where $E=L_{1}^{\vee} \oplus \cdots \oplus L_{n}^{\vee}$ is the Whitney sum of the tangent lines to the curve at its marked points. This means that the cohomology of the space $P \overline{\mathcal{H}}_{0, n}$ treated as an algebra over $H^{*}\left(\overline{\mathcal{M}}_{0, n}\right)$ are generated by the class $\psi=c_{1}(\mathcal{O}(1))$ subject to the relation

$$
\psi^{n}+c_{1}(E) \psi^{n-1}+\cdots+c_{n}(E)=0
$$

(cf. [16]). In particular, each class $\alpha$ in $H^{2 d}\left(P \overline{\mathcal{H}}_{0, n}\right)$ can be represented in the form

$$
\alpha=\pi^{*}\left(\eta_{d}\right)+\pi^{*}\left(\eta_{d-1}\right) \psi+\pi^{*}\left(\eta_{d-2}\right) \psi^{2}+\ldots
$$

where $\pi: P \overline{\mathcal{H}}_{0, n} \rightarrow \overline{\mathcal{M}}_{0, n}$ is the natural projection, and the classes $\eta_{i}$ belong to the cohomology of the space $\overline{\mathcal{M}}_{0, n}$. The well-known relation $\pi_{*} \psi^{s}=c_{s-n+1}(-E)$ (see, e.g., [3]) implies that the degree of any class represented in this form can be computed using the relation

$$
\operatorname{deg} \alpha=\int_{P \overline{\mathcal{H}}_{0, n}} \frac{\pi^{*}\left(\eta_{d}\right)+\pi^{*}\left(\eta_{d-1}\right) \psi+\ldots}{1-\psi}=\int_{\overline{\mathcal{M}}_{0, n}} \frac{\eta_{d}+\eta_{d-1}+\eta_{d-2}+\ldots}{c(E)} .
$$

This relation reduces the computation of the degree to a computation of some 
are well known (see, e.g., [2] or [14]). Below we represent the results of computations of the degrees of the pushforwarded basic classes up to codimension 2:

$$
\begin{aligned}
\operatorname{deg}(1) & =n^{n-3}, \\
\operatorname{deg}\left(\delta_{0,0}\right) & =\frac{1}{2}(n-1)(n+6) n^{n-4}, \\
\operatorname{deg}\left(\xi_{2}\right) & =\frac{1}{3}(n-1)\left(17 n^{2}-28 n+12\right) n^{n-5}, \\
\operatorname{deg}\left(\delta_{1,0}\right) & =-\frac{1}{6}(n-1)\left(n^{2}+10 n-120\right) n^{n-5}, \\
\operatorname{deg}\left(\delta_{0,0}^{2}\right) & =\frac{1}{12}(n-1)\left(3 n^{3}+31 n^{2}+82 n-120\right) n^{n-5} .
\end{aligned}
$$

Substituting these equations in the expressions for the strata we obtain the following formulas for their degrees:

$$
\begin{aligned}
\operatorname{deg}\left(\sigma_{2^{1}}\right) & =\frac{9}{2}(n-2)(n-1) n^{n-4}, \\
\operatorname{deg}\left(\sigma_{1^{2}}\right) & =2(n-3)(n-2)(n-1) n^{n-4}, \\
\operatorname{deg}\left(\sigma_{3^{1}}\right) & =\frac{32}{3}(n-3)(n-2)(n-1) n^{n-5}, \\
\operatorname{deg}\left(\sigma_{1^{1} 2^{1}}\right) & =9(n-4)(n-3)(n-2)(n-1) n^{n-5}, \\
\operatorname{deg}\left(\sigma_{1^{3}}\right) & =\frac{4}{3}(n-5)(n-4)(n-3)(n-2)(n-1) n^{n-5}, \\
\operatorname{deg}\left(\sigma_{2^{1}, 2^{1}}\right) & =\frac{3}{8}(n-2)(n-1)\left(27 n^{2}-137 n+180\right) n^{n-5}, \\
\operatorname{deg}\left(\sigma_{2^{1}, 1^{2}}\right) & =3(n-3)(n-2)(n-1)\left(3 n^{2}-15 n+20\right) n^{n-5}, \\
\operatorname{deg}\left(\sigma_{1^{2}, 1^{2}}\right) & =(n-3)(n-2)(n-1)\left(2 n^{3}-16 n^{2}+43 n-40\right) n^{n-5} .
\end{aligned}
$$

Now Theorem 1.1 produces the following formulas for the Hurwitz numbers:

$$
\begin{gathered}
h_{2^{1}}=\frac{9}{2} \frac{(2 n-4) !}{(n-3) !} n^{n-5}, \\
h_{1^{2}}=2 \frac{(2 n-4) !}{(n-4) !} n^{n-5}, \\
h_{3^{1}}=\frac{32}{3} \frac{(2 n-5) !}{(n-4) !} n^{n-6}, \\
h_{1^{1} 2^{1}}=9 \frac{(2 n-5) !}{(n-5) !} n^{n-6}, \\
h_{1^{3}}=\frac{4}{3} \frac{(2 n-5) !}{(n-6) !} n^{n-6}, \\
3 h_{2^{1}, 2^{1}}=\frac{3}{4}\left(27 n^{2}-137 n+180\right) \frac{(2 n-6) !}{(n-3) !} n^{n-6}, \\
h_{2^{1}, 1^{2}}=3\left(3 n^{2}-15 n+20\right) \frac{(2 n-6) !}{(n-4) !} n^{n-6}, \\
h_{1^{2} 1^{2}}=2\left(2 n^{3}-16 n^{2}+43 n-40\right) \frac{(2 n-6) !}{(} n^{n-6} ;
\end{gathered}
$$


the first group of formulas here refers to the case of a single degenerate ramification, and these formulas are special cases of the Hurwitz formula.

Another approach to the calculation of the degrees of the strata consists in the direct application of the Hurwitz formula (or, for an arbitrary genus, the formula from [2] which is, however, less explicit) for Hurwitz numbers of multisingularities. Theorem 1.1 allows one to find the degree of each stratum of the form $\sigma_{\alpha}$. In the rational case $g=0$ knowing the decompositions of the strata as polynomials in the pushforwards of basic classes we can compute the degrees of at least some of these direct images. In our case it is easy to compute the degrees $\operatorname{deg}(1), \operatorname{deg}\left(\delta_{0,0}\right)$, $\operatorname{deg}\left(\delta_{1,0}\right), \operatorname{deg}\left(\xi_{2}\right)$. Note, however, that knowing these degrees is insufficient for computing the degrees of the strata of multimultisingularities: their decomposition includes the class $\delta_{0,0}^{2}$. The degree of the latter can be computed, for example, by using the degree of the stratum $\sigma_{2^{1}, 2^{1}}$, which is known from [22].

4.3. On nonisolated singularities. Although we do not yet have a complete understanding of what is going on when nonisolated singularities are added to the picture, a few words about them are in order. Consider the first nontrivial contribution of nonisolated singularities in the case of rational functions, i.e., the classes of codimension 3. Denote by $\sigma_{\alpha_{1}, \alpha_{2}, \ldots}^{\exp }$ the expected decompositions for the corresponding classes obtained by the universal formulas under the assumption that there are no singularities of types other than $A_{k}$ and $I_{k, l}$. They differ from the correct decompositions of the classes $\sigma_{\alpha_{1}, \alpha_{2}, \ldots}$ by terms supported on the locus $I_{\infty} \subset B$ of functions with nonisolated singularities. Since the correction terms have the same dimension as the subvariety $I_{\infty}$, they must be proportional to the fundamental class of this subvariety, with a constant proportionality coefficient. It is natural to assume that this coefficient is universal, that is, it depends neither on $n$, nor on the choice of an irreducible component of the subvariety $I_{\infty}$. The following "unexpected" observation serves a numerical confirmation of this assumption: the degree of the difference

$$
\operatorname{deg}\left(\sigma_{\alpha_{1}, \alpha_{2}, \ldots}-\sigma_{\alpha_{1}, \alpha_{2}, \ldots}^{\exp }\right)
$$

is proportional to the degree of the stratum $I_{\infty}$,

$$
\operatorname{deg}\left(I_{\infty}\right)=\frac{1}{8}(n-1)\left(n^{3}+11 n^{2}+34 n-120\right) n^{n-5}
$$

with integer coefficients. The degrees of the classes $\sigma_{\alpha_{1}, \alpha_{2}, \ldots}^{\exp }$ can be computed by means of the methods described in the present paper, while the correct degree of the stratum $\sigma_{\alpha_{1}, \alpha_{2}, \ldots}$ can be deduced from the Hurwitz formula. Let us present the conjectural contribution of nonisolated singularities to some strata of codimension three:

$$
\begin{aligned}
\sigma_{4^{1}} & =\sigma_{4^{1}}^{\exp }+5 I_{\infty}, \\
\sigma_{1^{1} 3^{1}} & =\sigma_{1^{1} 3^{1}}^{\exp }-16 I_{\infty}, \\
\sigma_{2^{2}} & =\sigma_{2^{2}}^{\exp }-9 I_{\infty}, \\
\sigma_{1^{2} 2^{1}} & =\sigma_{1^{2} 2^{1}}^{\exp }+36 I_{\infty}, \\
\sigma_{1^{4}} & =\sigma_{1^{4}}^{\exp }-16 I_{\infty} .
\end{aligned}
$$




\section{REFERENCES}

1. Ekedahl T., Lando S. K., Shapiro M., Vainshtein A., On Hurwitz numbers and Hodge integrals, C. R. Acad. Sci. Paris. Sér. I. Math. 328 (1999), 1175-1180.

2. Ekedahl T., Lando S. K., Shapiro M., Vainshtein A., Hurwitz numbers and intersections on moduli spaces of curves, Invent. math. 146 (2001), 297-327.

3. Fulton W., Intersection Theory, Springer, Berlin, 1998.

4. Giusti M., Classification des singularités isolées simples d'intersections complètes, Proceedings of Symposia in Pure Mathematics. 40. Part 1 (1983), 457-494.

5. Goryunov V.V., Singularities of projections of complete intersections, Current Problems in Mathematics. Itogi Nauki i Techniki. Sovremennye Problemy Matematiki, Vol. 22, VINITI, Moscow, 1983, p. 167-206.

6. Goulden I. P., Jackson D. M., Transitive factorisation into transpositions and holomorphic mappings on the sphere, Proc. Amer. Math. Soc. 125 (1997), no. 1, 51-60.

7. Harris J., Mumford D., On the Kodaira dimension of the moduli space of curves, Invent. Math. 67 (1982), no. 1, 23-88.

8. Hurwitz A., Über Riemann'sche Flächen mit gegebenen Verzweigungpunkten, Math. Ann. 39 (1891), 1-61.

9. Hurwitz A., Über die Anzal der Riemann'sche Flächen mit gegebenen Verzweigungpunkten, Math. Ann. 55 (1902), 51-60.

10. Kazaryan M.E., Multisingularities, cobordisms, and enumerative geometry, Russ. Math. Surveys 58 (2003), no. 4, 665-724.

11. Kazaryan M.E., Morin maps and their characteristic classes. http://www.mi.ras.ru/kazaryan.

12. Kazaryan M. E., Classifying spaces of singularities and Thom polynomials, NATO Sci. Ser. II. Math. Phys. Chem. V.21. New developments in singularity theory, Kluwer Acad. Publ., Dordrecht, 2001, p. 117-134.

13. Kazaryan M.E., Relative Morse theory of one-dimensional foliations, and cyclic homology, Funct. Anal. Appl. 31 (1997), no. 1, 20-31.

14. Lando S.K., Ramified coverings of the two-dimensional sphere and the intersection theory in spaces of meromorphic functions on algebraic curves, Russ. Math. Surveys 57 (2002), no. 3, $463-533$.

15. Lando S.K., Zvonkine D.A., On multiplicities of the Lyashko-Looijenga mapping on the discriminant strata, Funct. Anal. Appl. 33 (1999), no. 3, 178-188.

16. Lando S. K., Zvonkine D., Counting ramified coverings and intersection theory on spaces of rational functions. I. math.AG/0303218.

17. Manin Yu., Zograf P., Invertible cohomological field theories and Weil-Petersson volumes, Ann. Inst. Fourier (Grenoble). 50 (2000), no. 2, 519-535.

18. Mumford D., Towards an enumerative geometry on the moduli spaces of curves, Progress in Math, vol. 36, Birkhüaser, Boston, 1983, p. 271-328.

19. Natanzon S.M., Turaev V., A compactification of the Hurwitz space, Topology. 38 (1999), 889-914.

20. Thom R., Quelque propriétés globales des variétés différentiables, Comment. Math. Helv. 28 (1954), 17-86.

21. Zvonkine D., Multiplicities of the Lyashko-Looijenga map on its strata, C. R. Acad. Sci. 324 (1997), Sér. I. 1349-1353.

22. Zvonkine D., Counting ramified coverings and intersection theory on Hurwitz spaces. II (Local structures of Hurwitz spaces and combinatorial results), Preprint, math.AG/0304251.

Steklov MAThematical Institute RAS AND

INDEPENDENT UNIVERSITY OF MOSCOW,

Institute FOR System Research RAS AND

INDEPENDENT UNIVERSITY OF MOSCOW, 